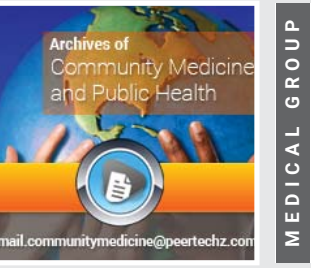

\section{Unravelling the impact of COVID-19 on mental health: A scoping review on traumatogenic events using the phases of response to disaster model}

\author{
Elke Van Hoof*\#, Hannah De Laet", Sophie Hochrath, Evelien \\ Philips, Paula Horczak and Nele Van den Cruyce \\ Vrije Universiteit Brussel, Department of Psychology, Faculty of Educational and Psychological Sciences, \\ Belgium \\ \#These authors contributed equally to this work
}

Received: 12 November, 2021

Accepted: 13 December, 2021

Published: 16 December, 2021

*Corresponding author: Elke Van Hoof, Vrije Universiteit Brussel, Department of Psychology, Faculty of Educational and Psychological Sciences, Belgium, |E-mail:Elke.van.hoof@vub.be

Keywords: COVID-19; Psychosocial impact; Pandemic preparedness; Mental health; Disaster response model

Copyright: (c) 2021 Van Hoof E, et al. This is an openaccess article distributed under the terms of the Creative Commons Attribution License, which permits unrestricted use, distribution, and reproduction in any medium, provided the original author and source are credited.

https://www.peertechzpublications.com

Check for updates

\title{
Abstract
}

Background: Disease outbreaks such as the COVID-19 pandemic give rise to high levels of psychological distress in people worldwide. Since this is the first pandemic of its kind, the best available evidence is needed on what psychological needs could be expected during and after the pandemic.

Objectives: In this scoping review existing research on traumatogenic events is examined in order to identify the potential impact on mental health of the COVID pandemic. The research findings are organized using the the phases of disaster response model.

Results: A total of 34 longitudinal studies, 2 studies with multiple waves of data collection and 92 cross-sectional studies met the inclusion criteria. The studies included in this scoping review could be classified as: 87 studies on COVID-19, 2 on SARS, 19 on wars, 19 on terrorist attacks and 1 on a nuclear accident. Results indicate that stress, anxiety, depressive symptoms, insomnia, denial, anger, grief and fear can be anticipated as common reactions. The longer a pandemic continues, the higher the psychological strain is expected to be.

Conclusions: The phases of response to disaster model offers a valid frame to unravel the impact of the pandemic on mental health over time. Specific attention must be given to vulnerable groups, whereby specific risk factors include age, gender, pre-existing mental health problems, healthcare profession, migration background, isolation and low socio economic status. However, these may change over time, and a delayed manifestation of psychosocial problems needs to be considered too. Mental health governance is, therefore, warranted throughout and even up to 6 months after the pandemic.

\section{Introduction}

The COVID-19 pandemic, caused by severe acute respiratory syndrome coronavirus 2 (SARS-CoV-2) has had an enormous impact on healthcare and economic systems but also on (public) mental health. The entire world has struggled with an emergency that has been unusually challenging, ubiquitous and cataclysmic for health and wellbeing [1], including a significant case fatality rate - albeit lower than SARS (Severe Acute Respiratory Syndrome) and MERS (Middle East respiratory Coronavirus syndrome) [2]. To date, with more than 14 million infected worldwide and a spread that is far from contained, monitoring the psychological impact of this pandemic on the general population as well as in atrisk groups has become increasingly important [2]. While the context and the specific approaches for dealing with the pandemic vary across the globe, there are at least two common denominators that make the pandemic a traumatic experience for all involved. Firstly, people are confronted with serious illness on a vast scale and also with (mostly untimely and sudden) death, both of which are linked to an array of longlasting negative psychological effects [3]. Secondly, some 
degree of confinement measures have been adopted to stop the virus from spreading. COVID-19 is not only a highly contagious disease that has spread over vast areas, but it also took the world by storm, which necessitated drastic measures. The DSM-5 [4] indicates that "experiencing repeated or extreme exposure to aversive details of the traumatic event(s)" can be considered as a distinct type of exposure to trauma (Criterion A4). Indeed, one third of the world's population was subjected to varying degrees of lockdown, whereby society needed to abide by an array of different restrictive measures. Some lockdowns included a total movement control or stay-at-home order, only leaving essential shops open, whilst others consisted of more limited and time-bound restrictions, for example, a curfew. A significant number of people also experienced quarantine, which involves separating out and restricting the movement of those people who have potentially and/or effectively been exposed to COVID-19 in order to stop the virus from spreading further [5].

Traumatic experiences are known to have an impact on body, mind and relationships with others, sometimes even over a longer period of time. According to DeWolfe [6] there are two types of trauma: individual, and community or universal trauma. While individual trauma focuses on the traumatic experience - both singular and multiple or chronic events of an individual (ex. robbery, rape, life threatening illness, ...), community trauma involves entire communities and is, thereby, characterized by mutual recognition and shared experience within a group of people, which can often put a strain on social support systems [7]. This latter kind of trauma is common in disasters (e.g., mass shooting, war, flooding, ...), in which anyone who has lived through the disaster is at risk of experiencing trauma-related reactions. It is, therefore, also referred to as a universal trauma, breaching the generally accepted norms within a society, although the specific impact of the trauma on the people involved may differ based on characteristics of the individual, the type and characteristics of the disaster, developmental processes, and sociocultural factors. Direct exposure and proximity to a disaster are generally linked to an increased risk of traumatic impact [8]. All these elements create groups of people at different levels of risk, for example, direct victims, families of victims, carers, and so forth. COVID-19 has all the characteristics of a universal trauma. Nevertheless, it remains unclear what can be expected of the specific impact on the psychosocial needs within society, since it is the first contagious outbreak of its kind.

COVID-19 is, indeed, the first major disaster of this amplitude in the $21^{\text {st }}$ century. Emergency and/or pandemic preparedness is, currently, strongly focused on fast-burning [9] or situational crises [10] characterized by an immediate threat which - if responded to appropriately - is of a shortterm nature. The current strategies for emergency and/or pandemic planning and response are, thereby, anchored in "policy as routine" [11] contributing to homeostasis: a known intervention when a sudden anomaly occurs in a structured world or rather clearly delineated system (e.g., a factory fire, flooding of a village), with the purpose of fixing the problem, after which the situation returns to the norm and business continues as usual (apart from minor adjustments based on incremental learning). COVID-19 challenges the homeostasis principle within society, because there is no standard fix or readily available intervention for this kind of complex challenge that could serve to quickly restore society's stability, but rather it is chronic in nature. While this is true for society and emergency and/or pandemic preparedness in general, it is particularly applicable to the specific domain of psychosocial health.

The current scoping review aims to create order in chaos and uncertainty, by better understanding what can be expected regarding the psychosocial impact of COVID-19 looking at it as a disaster and traumatogenic experience. A theoretical model that can be of use when looking into the possible psychosocial impact of disasters on people is the phases of response to disaster model [12]

According to DeWolfe [6] a disaster can be divided into seven phases. It starts with the threat or warning phase, which refers to the time before a potential disaster. Responses to a warning or threat of a disaster vary from active response to ignoring the warning. While ignoring a warning may lead to feelings of guilt, receiving no warning at all may result in feelings of vulnerability and fearfulness [6]. Accurate information about what to expect and how to act must be provided by trustworthy and recognized authorities [13]. The moment of the disaster is called the impact phase. The specific impact on the individual and the community depends on the type and severity of the disaster. Common responses are confusion, disbelief, shock or panic. The time immediately after the disaster is referred to as the rescue or heroic phase, in which survival and safety are the steering concepts. Levels of altruism, but also anxiety are high [6]. In this phase it is important for members of the community to help each other, to form groups and to take on active roles to reduce feelings of helplessness and powerlessness. Furthermore, it is important for authorities to make psychological first aid available, estimate shortand long-term effects and to arrange for human and other resources [13]. Giving and receiving help; and bonding with the community results in the remedy and honeymoon phase in which individuals tend to feel optimistic. Next, in the inventory phase, people reflect upon what happened. It is in this moment that the extent of losses and the limits of received or perceived lack of assistance may become clear, and unrealistic optimism can give way to exhaustion and discouragement. This phase is, thereby, followed by the disillusionment phase, in which individuals are prone to chronic stress because of the number and variety of stressors they face, while bonds with the community tend to weaken [6]. This phase is also referred to as the second disaster due to its negative effect on individuals' emotional well-being, which makes it important to further strengthen social networks and enable individuals to take on an active role. In addition, it is important that authorities provide practical and psychological support and set up communication systems to correct and prevent circulation of myths and other false information [13]. These interventions continue to be required during the final phase [13], the reconstruction or 
recovery phase, in which the recovery of physical and emotional well-being, growth and opportunity are central concepts [6].

The phases of response to disaster model has been validated in data-driven studies $[7,14]$. The timing of the different phases and the specific transition between the phases is, however, less well-defined and likely depends on the type of disaster in question. This is also seen in crisis management, where several authors refrain from using "phases" and instead use "stages", since there is an increasing recognition that there can and even should be a considerable time overlap in order to manage the disaster as well as possible $[15,16]$. In the case of COVID-19, society is currently confronted with a contagious disease, spreading around the world with a highly visible impact and a realistic risk of reoccurrence. The pandemic, therefore, features several factors known to prolong and worsen the psychological implications of a disaster [6,7]. The specific experience and impact of a disaster can, logically, differ amongst individuals and subgroups. However, although disasters are not uniform events with guaranteed universal, standardized experiences; the macro perspective provided by the phases of response to disaster model can help to facilitate community preparedness, development of interventions and, in this specific case, organization of research findings over time.

Therefore, this scoping review will use the phases of response to disaster model in order to define and organize specific mental health needs within society over time in response to a contagious disease outbreak and to analyze any differences from the currently available clinical knowledge on acute disasters in delineated communities. As this review will also aim to identify specific groups at higher risk of developing mental health problems, it can also offer useful insights to policymakers and mental health practitioners in the field who are attempting to provide effective and efficient psychological support for the public, especially where and when it is needed the most.

\section{Method}

The current report was written using the Preferred Reporting Items for Systematic Reviews and Meta-Analyses Extension for Scoping Reviews [17].

\section{Eligibility criteria}

Studies were included if they reported on the impact on mental health of a chronic and/or recurrent traumatogenic event, such as an infectious disease outbreak, war, or technological disaster. A traumatogenic event was defined as an occurrence in which people were confronted with the finite nature of life. Events with a chronic effect and/or a significant ripple effect were included. Single events, such as fireworks explosions, were not considered eligible. Only studies examining healthy populations, that is, no diagnosis of mental/behavioral disorders or severe medical problems, were included in the review. For cross-sectional studies, only reports with a total number of participants of $\geq 1000$ were included. In order to ensure the highest quality of evidence, only original articles were included. Additional inclusion criteria were: peerreviewed journal articles published in English with availability of the full text. Research describing the impact of natural disasters, intervention studies, expert opinions, letters to the editor and (systematic) reviews were excluded Figure 1.

\section{Information sources and search methods}

Two electronic databases were searched from January 2000 to July 11, 2021: PubMed and PsycInfo. The search strategy was designed by E.V.H. and refined and implemented by P.H and E.P. The final search query for PubMed can be found in supplementary material. The search results from the two databases were exported to EndNote and duplicate articles were removed.

\section{Selection of sources of evidence}

The flowchart of the scoping review process is shown

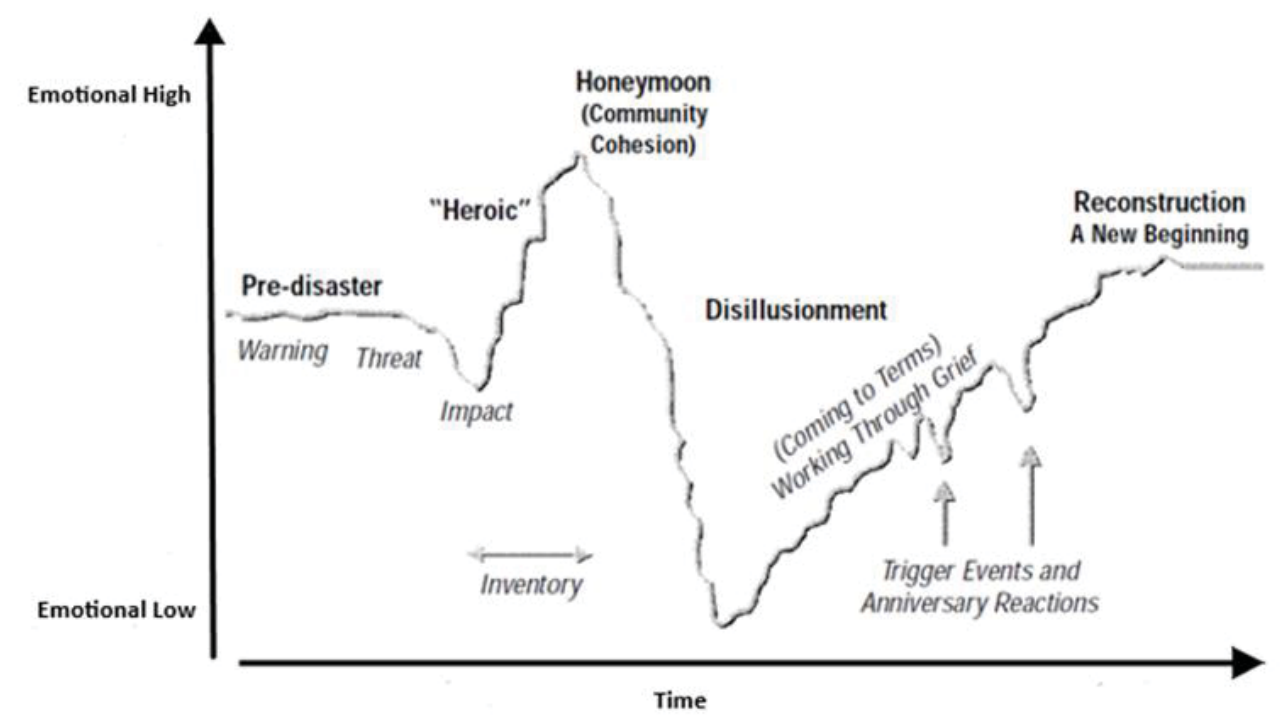

Citation: Van Hoof E, Laet HD, Hochrath S, Philips E, Horczak P, et al. (2021) Unravelling the impact of COVID-19 on mental health: A scoping review on traumatogenic events using the phases of response to disaster model. Arch Community Med Public Health 7(3): 218-237. DOI: https://dx.doi.org/10.17352/2455-5479.000165 


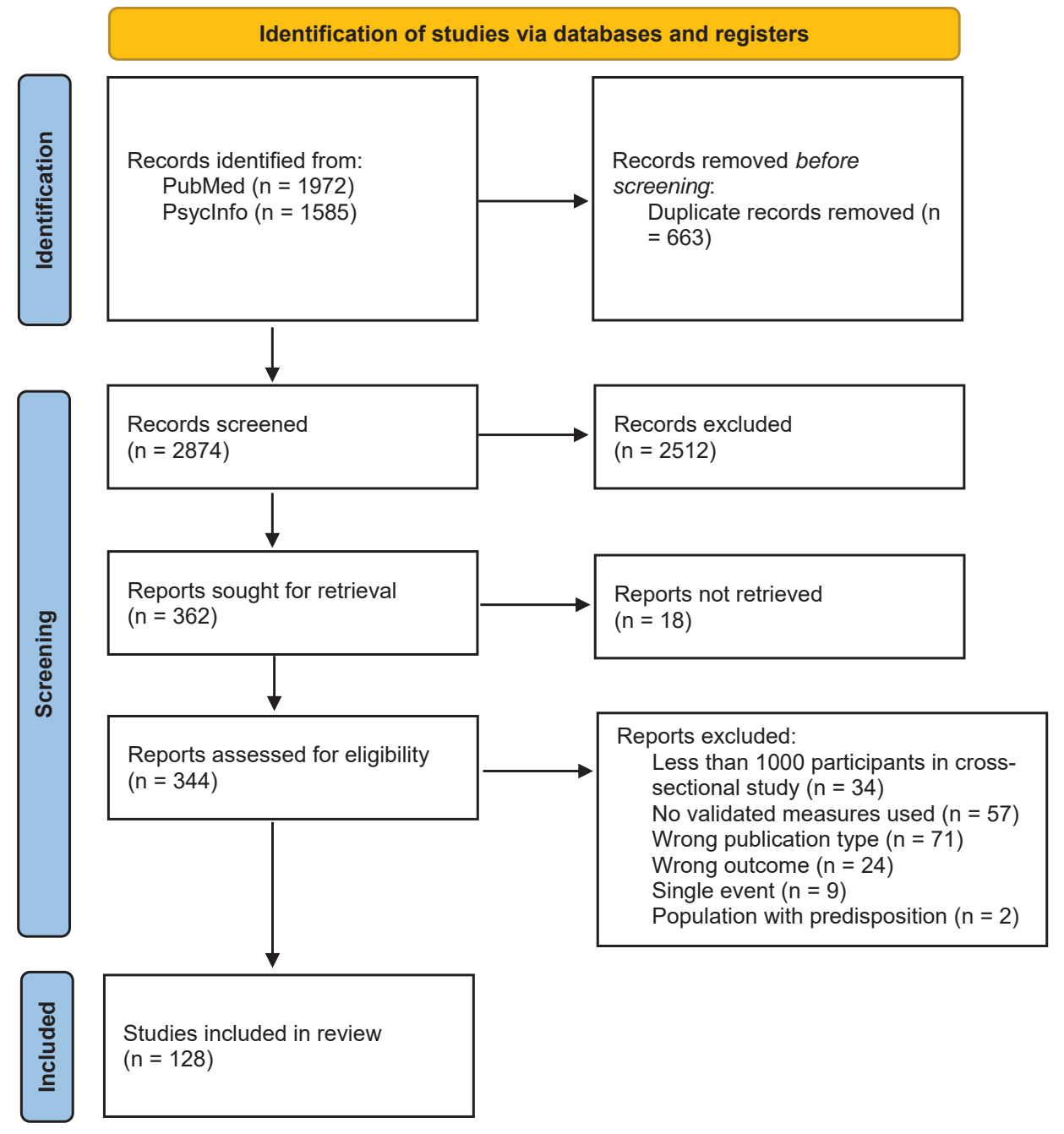

Figure 2: Flow-chart of the scoping review process.

in Figure 2. As a first step, two authors, H.D.L. and S.H., independently screened the articles for eligibility based on title and abstract. Articles which did not meet inclusion criteria were excluded from the review. Subsequently, the full texts of the remaining articles were retrieved and four reviewers, working in pairs, evaluated the records. Disagreements in one pair were resolved through discussion and consensus with a reviewer from another pair.

\section{Data extraction}

After analyzing the full texts, two main categories of studies were created: COVID-19-related studies and other disasterrelated studies. The following data were extracted: first author, year of publication, study design, sample size, mean age of participants, country, date of study/study duration, outcome variables related to common mental health disorders (such as anxiety, PTSD) and to coping, types of questionnaires used (for a detailed overview, see Table 1). Since the current review aimed to attain the highest level of evidence, only reports using a solid research methodology (i.e., using validated measures), were considered.

\section{Synthesis of results}

In the first part of the review, an overview of the findings from COVID-19 research conducted in the last year will be presented. This information can give us an impression of the initial impact of COVID-19 on mental health and identify specific groups at risk of developing mental health problems. The second part of the review organizes the remainder of the studies according to the phases of response to disaster model, enabling predictions of expected mental health impacts of COVID-19 over time.

\section{Results}

\section{Selection and characteristics of sources of evidence}

An overview of the study selection process is displayed in Figure 2. Of the 3,557 articles identified for this review, only 128 were included. A total of 34 longitudinal studies, 2 studies with multiple waves of data collection and 92 cross-sectional studies met the inclusion criteria for this scoping review. The included research comprised 87 studies on COVID-19, 2 on SARS, 19 on war, 2 on terrorist attacks and 1 on a nuclear 
Table 1: Characteristics of included studies.

\begin{tabular}{|c|c|c|c|c|c|c|c|c|}
\hline $\begin{array}{c}\text { First author (year of } \\
\text { publication) }\end{array}$ & $\mathbf{N}$ & $\begin{array}{l}\text { Mean age } \\
\text { (SD) }\end{array}$ & Country & $\begin{array}{c}\text { Date/ } \\
\text { duration }\end{array}$ & Event & Design & Outcome & Questionnaire \\
\hline $\begin{array}{l}\text { Achterberg, et al. } \\
{[87]}\end{array}$ & 257 & & $\begin{array}{l}\text { The } \\
\text { Netherlands }\end{array}$ & & COVID-19 & Longitudinal & $\begin{array}{l}\text { Depression, anxiety, } \\
\text { stress, coping }\end{array}$ & BSI, PSS-10, CERQ \\
\hline Büssing, et al. [20] & 1277 & $50.9(14.9)$ & Germany & June $9-21,2020$ & COVID-19 & $\begin{array}{l}\text { Cross-sectional } \\
\text { online survey }\end{array}$ & Wellbeing & WHO-5 \\
\hline \multirow[t]{2}{*}{ Banerjee, et al. [86] } & 1520 & & $\begin{array}{l}101 \text { countries, } \\
\text { worldwide }\end{array}$ & April - May, 2020 & COVID-19 & $\begin{array}{l}\text { Cross-sectional } \\
\text { online survey }\end{array}$ & Wellbeing & eWBI \\
\hline & 272 & & & July - August, 2020 & & $\begin{array}{l}\text { Follow up of the } \\
\text { first survey }\end{array}$ & & \\
\hline Barzilay, et al. [18] & 1350 & $41(13)$ & USA & April $6-15,2020$ & COVID-19 & $\begin{array}{l}\text { Cross-sectional } \\
\text { online survey }\end{array}$ & Anxiety, depression & GAD-7, PHQ-2 \\
\hline \multirow[t]{3}{*}{ Ben-Zur, et al. [104] } & 701 & & Israel & $\begin{array}{l}\text { T1: } 26 \text { - } 34 \text { days before } \\
\text { the event of the disen- } \\
\text { gage-ment, which took } \\
\text { place during August } 15 \\
\quad-22,2005\end{array}$ & $\begin{array}{c}\text { Disengagement from } \\
\text { Gaza and Second } \\
\text { Lebanese War }\end{array}$ & $\begin{array}{l}\text { Longitudinal; } \\
\text { telephone } \\
\text { interviews }\end{array}$ & Anxiety & BSI \\
\hline & 462 & & & $\begin{array}{l}\text { T2: } 21 \text { - } 41 \text { days after } \\
\text { the event of the disen- } \\
\text { gagement }\end{array}$ & & & & BSI \\
\hline & 366 & & & $\begin{array}{l}\text { T3: } 19 \text { - } 37 \text { days after } \\
\text { the end of the Second } \\
\text { Lebanese War, which } \\
\text { took place } \\
\text { between July } 12 \text { and } \\
\text { August } 14,2006\end{array}$ & & & PTSD & BSI, PSS-SR \\
\hline $\begin{array}{l}\text { Birkeland, et al. } \\
\qquad[105]\end{array}$ & 256 & & Norway & $\begin{array}{c}\text { April - May, 2012, } 2013 \\
\text { and } 2014\end{array}$ & Oslo bombing & Longitudinal & PTSD & PCL \\
\hline $\begin{array}{l}\text { Birkeland, et al. } \\
\text { [105] }\end{array}$ & 1970 & & Norway & April 2012 & Oslo bombing & $\begin{array}{c}\text { Cross-sectional } \\
\text { survey }\end{array}$ & PTSD & PCL \\
\hline $\begin{array}{l}\text { Birkeland, et al. } \\
\qquad[106]\end{array}$ & 1970 & & Norway & April 2012 & Oslo bombing & $\begin{array}{c}\text { Cross-sectional } \\
\text { survey }\end{array}$ & Depression, anxiety & SCL \\
\hline Biron, et al. [136] & 2072 & & Israel & $\begin{array}{l}\text { July } 23 \text {-August 10, } \\
\qquad 2006\end{array}$ & Second Lebanon war & $\begin{array}{l}\text { Longitudinal; } \\
\text { telephone } \\
\text { interviews }\end{array}$ & Negative affect & PANAS \\
\hline Blix, et al. [108] & 229 & & Norway & April - May, 2012, 2013 & Oslo bombing & Longitudinal & PTSD & PCL-S \\
\hline Bowler, et al. [109] & 4017 & & USA & $\begin{array}{l}\text { Septem-ber } 2003 \text { - } \\
\text { Novem-ber } 2004\end{array}$ & $\begin{array}{l}\text { Terrorist attacks of } \\
\qquad 9 / 11\end{array}$ & $\begin{array}{l}\text { Cross-sectional; } \\
\text { telephone } \\
\text { interview }\end{array}$ & PTSD & PCL \\
\hline Butler, et al. [131] & 1281 & & USA & $\begin{array}{l}\text { T1: Septem-ber } 21 \text { - } \\
\text { December 4, } 2001 \\
\text { T2: March } 12 \text { - May 19, } \\
2002\end{array}$ & $\begin{array}{l}\text { Terrorist attacks of } \\
\qquad 9 / 11\end{array}$ & $\begin{array}{l}\text { Longitudinal; } \\
\text { online survey }\end{array}$ & $\begin{array}{l}\text { Wellbeing, global } \\
\text { distress, coping }\end{array}$ & $\begin{array}{c}\text { Scales of } \\
\text { Psychological Well- } \\
\text { Being, BSI, Brief } \\
\text { COPE }\end{array}$ \\
\hline Cai, et al. [21] & 1521 & & China & & COVID-19 & Cross-sectional & $\begin{array}{l}\text { Depression, anxiety, } \\
\text { resilience }\end{array}$ & SLC-90, CD-RISC \\
\hline Cardeña, et al. [135] & 3134 & 43.1 & USA & $\begin{array}{l}\text { Septem-ber } 20 \text { - } \\
\text { October 4, } 2001\end{array}$ & $\begin{array}{l}\text { Terrorist attacks of } \\
9 / 11\end{array}$ & $\begin{array}{l}\text { Cross-sectional; } \\
\text { online survey }\end{array}$ & $\begin{array}{c}\text { Post-traumatic reactions, } \\
\text { coping }\end{array}$ & SASRQ, Brief COPE \\
\hline Carriedo, et al. [22] & 1795 & $\begin{array}{c}40.54 \\
(15.68)\end{array}$ & Spain & March 21 - 25, 2020 & COVID-19 & $\begin{array}{l}\text { Cross-sectional; } \\
\text { online survey }\end{array}$ & Resilience & CD-RISC \\
\hline $\begin{array}{l}\text { Cauberghe, et al. } \\
\text { [23] }\end{array}$ & 2165 & $\begin{array}{l}15.51 \\
(1.59)\end{array}$ & Belgium & April $16-30,2020$ & COVID-19 & $\begin{array}{l}\text { Cross-sectional; } \\
\text { online survey }\end{array}$ & Anxiety, coping & GAD-7, Brief COPE \\
\hline Cheng, et al. [69] & 1270 & & UK and USA & $\begin{array}{l}\text { T1: March } 16-22,2020 \\
\text { T2: May } 18-24,2020\end{array}$ & COVID-19 & $\begin{array}{l}\text { Longitudinal; } \\
\text { online survey }\end{array}$ & Generic anxiety & STAI-Y1 \\
\hline Cheng, et al. [69] & 1047 & & UK and USA & $\begin{array}{l}\text { T1: March } 16-22,2020 \\
\text { T2: May } 18-24,2020\end{array}$ & COVID-19 & $\begin{array}{l}\text { Longitudinal; } \\
\text { online survey }\end{array}$ & Coping, depression & Brief COPE, CES-D \\
\hline Chen, et al. [24] & 12596 & $33.1(7.5)$ & $\begin{array}{l}\text { China and } \\
\text { Taiwan }\end{array}$ & April 2020 & COVID-19 & $\begin{array}{l}\text { Cross-sectional; } \\
\text { (online) survey }\end{array}$ & Trauma, burnout & TSQ, MBI-GS \\
\hline Chen, et al. [24] & 18171 & & $\begin{array}{l}35 \text { countries, } \\
\text { world-wide }\end{array}$ & April $9-20,2020$ & COVID-19 & $\begin{array}{l}\text { Cross-sectional; } \\
\text { online survey }\end{array}$ & $\begin{array}{l}\text { Depression, psycho- } \\
\text { logical distress }\end{array}$ & DASS-21, K6 \\
\hline Chong, et al. [27] & 9565 & & $\begin{array}{l}78 \text { countries, } \\
\text { world-wide }\end{array}$ & April - June, 2020 & COVID-19 & $\begin{array}{l}\text { Cross-sectional; } \\
\text { online survey }\end{array}$ & Coping & Brief COPE \\
\hline Cénat, et al. [79] & 1267 & & $\begin{array}{c}\text { Haiti, DRC, } \\
\text { Rwanda, Togo }\end{array}$ & March - May 2020 & COVID-19 & Cross-sectional & Anxiety, resilience & SCL-A, CD-RISC2 \\
\hline Daly, et al. [89] & 7319 & & USA & March 10 -July 20, 2020 & COVID-19 & Longitudinal & Psycho-logical distress & PHQ-2 \\
\hline Daly, et al. [90] & 10918 & & UK & $\begin{array}{c}\text { 2019, April - } \\
\text { September, } 2020\end{array}$ & COVID-19 & Longitudinal & Psycho-logical distress & GHQ-12 \\
\hline
\end{tabular}




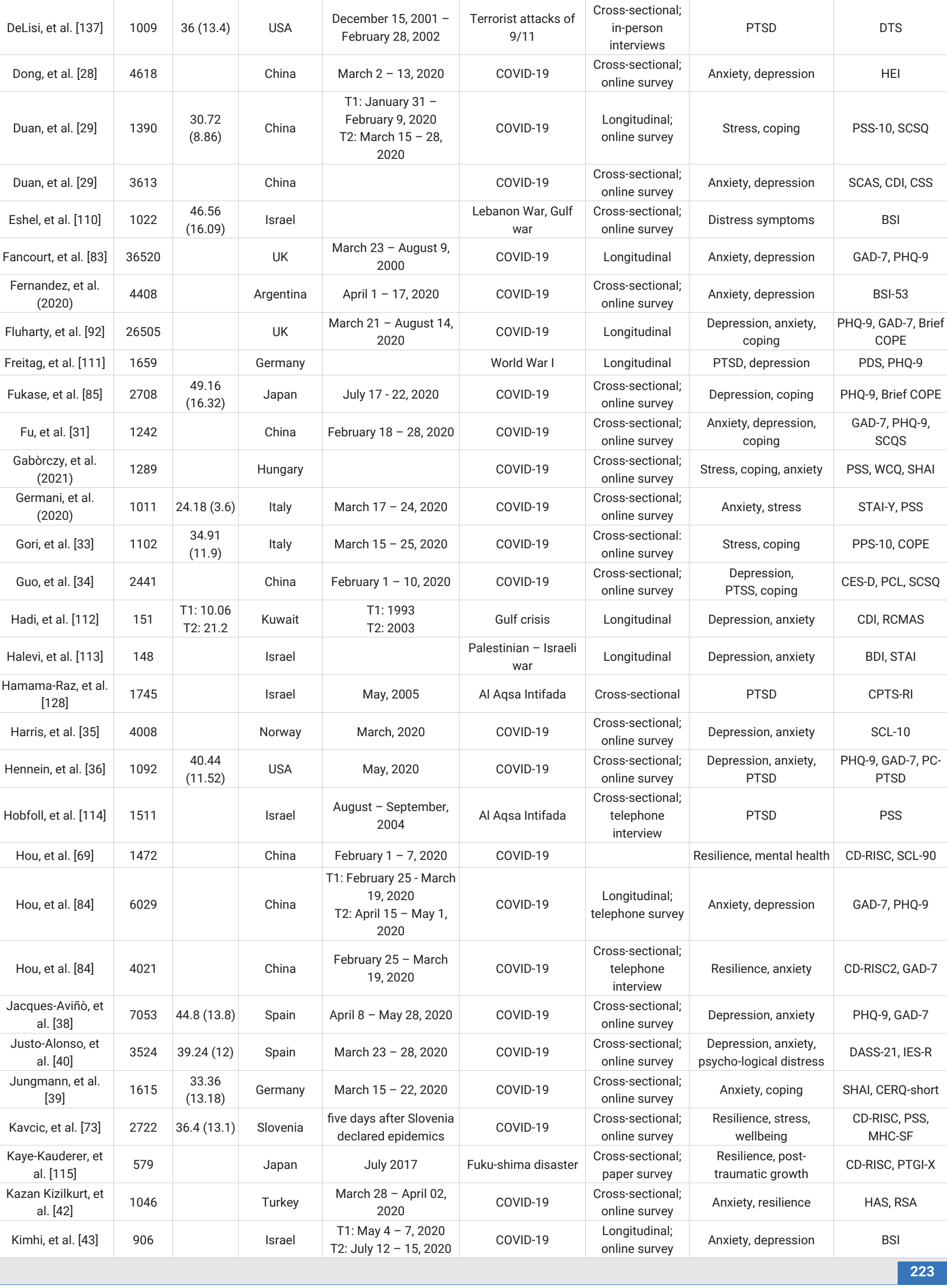




\begin{tabular}{|c|c|c|c|c|c|c|c|c|}
\hline Kimhi, et al. [93] & 1346 & $42(16.35)$ & Israel & & COVID-19 & $\begin{array}{l}\text { Cross-sectional; } \\
\text { online survey }\end{array}$ & $\begin{array}{l}\text { Anxiety, depression, } \\
\text { resilience }\end{array}$ & BSI, CD-RISC10 \\
\hline Koh, et al. [130] & 15025 & & Singapore & & SARS & $\begin{array}{l}\text { Cross-sectional; } \\
\text { paper survey }\end{array}$ & Psychological distress & IES \\
\hline Kung, et al. [116] & 33886 & & USA & $\begin{array}{l}\text { T1: 2003-2004 } \\
\text { T2: 2006-2007 }\end{array}$ & $\begin{array}{l}\text { Terrorist attacks of } \\
9 / 11\end{array}$ & Longitudinal & PTSD & PCL-C \\
\hline $\begin{array}{l}\text { Kuterovac-Jagodic, } \\
\text { et al. [117] }\end{array}$ & 252 & $10.2(1.19)$ & Croatia & $\begin{array}{c}\text { T1: December, } 1994 \\
\text { T2: May, } 1997\end{array}$ & Yougoslav wars & Longitudinal & $\begin{array}{l}\text { Post-traumatic stress } \\
\text { reactions, war traumata } \\
\text { and stressors, coping }\end{array}$ & $\begin{array}{l}\text { QPTSR-C, QSTWE, } \\
\text { SCSI-R }\end{array}$ \\
\hline Lahiri, et al. [44] & 1249 & & India & April 17 - May 16, 2020 & COVID-19 & $\begin{array}{l}\text { Cross-sectional; } \\
\text { online survey }\end{array}$ & $\begin{array}{c}\text { Coping, psycho-logical } \\
\text { distress }\end{array}$ & BRCS, K6 \\
\hline $\begin{array}{l}\text { Le Vigouroux, et } \\
\text { al. [45] }\end{array}$ & 1297 & $\begin{array}{l}21.27 \\
(4.72)\end{array}$ & France & April 23 - May 8, 2020 & COVID-19 & $\begin{array}{l}\text { Cross-sectional; } \\
\text { online survey }\end{array}$ & $\begin{array}{l}\text { Anxiety, depression, } \\
\text { coping }\end{array}$ & HADS, Brief COPE \\
\hline Levine, et al. [118] & $\begin{array}{l}\text { T1: } 2908 \\
\text { T2: } 588\end{array}$ & & Israel & $\begin{array}{c}\text { T1: } ? \\
\text { T2: } 1 \text { year after Second } \\
\text { Lebanon War }\end{array}$ & Second Lebanon War & Longitudinal & PTS & CPTS-RI, PCL \\
\hline Li, et al. [46] & 23192 & $\begin{array}{c}41.58 \\
(14.63)\end{array}$ & China & $\begin{array}{c}\text { March } 25-\text { April 1, } \\
2020\end{array}$ & COVID-19 & $\begin{array}{l}\text { Cross-sectional; } \\
\text { online survey }\end{array}$ & Resilience & CD-RISC2 \\
\hline Li, et al. [1] & 1109 & & China & March, 2020 & COVID-19 & $\begin{array}{l}\text { Cross-sectional; } \\
\text { online survey }\end{array}$ & $\begin{array}{l}\text { Anxiety, depression, } \\
\text { PTSD, coping }\end{array}$ & $\begin{array}{c}\text { GHQ-28, IES-R-22, } \\
\text { Brief COPE }\end{array}$ \\
\hline Li, et al. [46] & 2640 & & China & February $21-24,2020$ & COVID-19 & $\begin{array}{l}\text { Cross-sectional; } \\
\text { online survey }\end{array}$ & Coping, anxiety & CSQ, SAS \\
\hline Lorenzo, et al. [94] & $291+$ & & USA & $\begin{array}{l}\text { T1: April } 20-\text { May 15, } \\
2020 \\
\text { T2: one month after T1 }\end{array}$ & COVID-19 & Longitudinal & $\begin{array}{l}\text { Anxiety, depression, } \\
\text { coping }\end{array}$ & $\begin{array}{c}\text { GAD-7, PHQ-8, Brief } \\
\text { COPE }\end{array}$ \\
\hline $\begin{array}{l}\text { Luceño-Moreno, et } \\
\text { al. [47] }\end{array}$ & 1422 & $\begin{array}{c}43.88 \\
(10.82)\end{array}$ & Spain & April $1-30,2020$ & COVID-19 & $\begin{array}{l}\text { Cross-sectional } \\
\text { online survey }\end{array}$ & $\begin{array}{l}\text { PTSD, depression, } \\
\text { anxiety, burnout, } \\
\text { resilience }\end{array}$ & $\begin{array}{c}\text { IES-R, HADS, MBI, } \\
\text { BRS }\end{array}$ \\
\hline $\begin{array}{c}\text { Makhashvili, et al. } \\
\text { [48] }\end{array}$ & 2088 & & $\begin{array}{l}\text { Republic of } \\
\text { Georgia }\end{array}$ & May 25 - June 25, 2020 & COVID-19 & $\begin{array}{l}\text { Cross-sectional; } \\
\text { online survey }\end{array}$ & $\begin{array}{c}\text { Anxiety, depression, } \\
\text { PTSD }\end{array}$ & GAD-7, PHQ-9, ITQ, \\
\hline Marchetti, et al. [49] & 1226 & & Italy & April $3-14,2020$ & COVID-19 & $\begin{array}{l}\text { Cross-sectional; } \\
\text { online survey }\end{array}$ & $\begin{array}{l}\text { Psycho-logical distress, } \\
\text { resilience }\end{array}$ & GHQ-12; PAPF-PR \\
\hline $\begin{array}{l}\text { Martinez-Cao, et al. } \\
\qquad(2021)\end{array}$ & 21207 & $39.7(14)$ & Spain & March $19-26,2020$ & COVID-19 & $\begin{array}{l}\text { Cross-sectional; } \\
\text { online survey }\end{array}$ & Depression, anxiety & DASS-21, IES \\
\hline Matt, et al. [119] & 7605 & & USA & $\begin{array}{l}\text { Mid February, Mid } \\
\text { Septem-ber, 2000, } \\
\text { 2001, } 2002\end{array}$ & $\begin{array}{l}\text { Terrorist attacks of } \\
9 / 11\end{array}$ & Longitudinal & $\begin{array}{c}\text { Depression, anxiety, } \\
\text { PTSD }\end{array}$ & BDI, STAI, PCL-C \\
\hline $\begin{array}{l}\text { McCracken, et al. } \\
{[50]}\end{array}$ & 1102 & & Sweden & $\begin{array}{c}\text { May } 14^{\text {th }}-\text { June } 11 \\
2020\end{array}$ & COVID-19 & $\begin{array}{l}\text { Cross-sectional; } \\
\text { online survey }\end{array}$ & Depression, anxiety & PHQ-9, GAD-7 \\
\hline $\begin{array}{l}\text { McFadden, et al. } \\
\text { [51] }\end{array}$ & 3425 & & UK & May 7 - July 3, 2020 & COVID-19 & $\begin{array}{l}\text { Cross-sectional; } \\
\text { online survey }\end{array}$ & Wellbeing, coping & $\begin{array}{l}\text { SWEMWBS, Brief } \\
\text { COPE }\end{array}$ \\
\hline $\begin{array}{c}\text { Mclntosh, et al. } \\
{[133]}\end{array}$ & & & USA & $\begin{array}{c}\text { June, } 2001 \text { - Novem- } \\
\text { ber, } 2004\end{array}$ & $\begin{array}{l}\text { Terrorist attacks of } \\
9 / 11\end{array}$ & $\begin{array}{l}\text { Longitudinal; } \\
\text { online survey }\end{array}$ & PTS & IES-R, PCL \\
\hline Mechili, et al. [52] & 1112 & & Albania & $\begin{array}{c}\text { March } 30-\text { April 9, } \\
2020\end{array}$ & COVID-19 & $\begin{array}{l}\text { Cross-sectional; } \\
\text { online survey }\end{array}$ & Depression & PHQ-9 \\
\hline $\begin{array}{l}\text { Mikocka-Walus, et } \\
\text { al. [100] }\end{array}$ & 2110 & & Australia & & COVID-19 & Cross-sectional & $\begin{array}{l}\text { Depression, anxiety, } \\
\text { stress, resilience }\end{array}$ & DASS-21, BRS \\
\hline Minahan, et al. [53] & 1318 & $\begin{array}{c}52.42 \\
(17.76)\end{array}$ & USA & April 1 - May 17, 2020 & COVID-19 & $\begin{array}{l}\text { Cross-sectional; } \\
\text { online survey }\end{array}$ & $\begin{array}{l}\text { Post-traumatic stress, } \\
\text { coping, depression, } \\
\text { anxiety }\end{array}$ & $\begin{array}{l}\text { IES-R, Brief COPE, } \\
\text { PHQ-9, GAD-7 }\end{array}$ \\
\hline Mosheva, et al. [55] & 1106 & & Israel & March $19-22,2020$ & COVID-19 & $\begin{array}{l}\text { Cross-sectional; } \\
\text { online survey }\end{array}$ & Resilience & CD-RISC10 \\
\hline $\begin{array}{l}\text { Mohd Fauzi, et al. } \\
\text { [54] }\end{array}$ & 1050 & $\begin{array}{l}33.08 \\
(6.97)\end{array}$ & Malaysia & May, 2020 & COVID-19 & $\begin{array}{l}\text { Cross-sectional; } \\
\text { online survey }\end{array}$ & Depression, anxiety & DASS-21 \\
\hline Morina, et al. [120] & 3313 & 42.3 & $\begin{array}{c}\text { Bosnia } \\
\text { Herzegovina, } \\
\text { Croatia, } \\
\text { Kosovo, } \\
\text { Macedonia, } \\
\text { and Serbia }\end{array}$ & $2006-2007$ & Yougoslav wars & Longitudinal & Depression, PTSD & MINI, BSI, IES-R \\
\hline $\begin{array}{c}\text { Moya-Lacasa, et } \\
\text { al. [80] }\end{array}$ & 21152 & $\begin{array}{c}39.75 \\
(14.04)\end{array}$ & Spain & March $19-26,2020$ & COVID-19 & $\begin{array}{l}\text { Cross-sectional; } \\
\text { online survey }\end{array}$ & Depression, anxiety & DASS-21, IES \\
\hline Nissen, et al. [140] & 1881 & $45.4(10.9)$ & Norway & $\begin{array}{c}9-10 \text { months after the } \\
\text { terrorist attack }\end{array}$ & Oslo bombing & Cross-sectional & PTSD & PCL \\
\hline & & & & & & & & 224 \\
\hline
\end{tabular}

Citation: Van Hoof E, Laet HD, Hochrath S, Philips E, Horczak P, et al. (2021) Unravelling the impact of CoVID-19 on mental health: A scoping review on traumatogenic events using the phases of response to disaster model. Arch Community Med Public Health 7(3): 218-237. DOI: https://dx.doi.org/10.17352/2455-5479.000165 


\begin{tabular}{|c|c|c|c|c|c|c|c|c|}
\hline $\begin{array}{c}\text { Nuttman-Shwartz, et } \\
\text { al. (2018) }\end{array}$ & 1054 & & Israel & 2012 & $\begin{array}{c}\text { Missile attacks and } \\
\text { military operation } \\
\text { between Israel and } \\
\text { Gaza }\end{array}$ & Cross-sectional & Resilience, stress & CYRM-28, BSI \\
\hline Palmieri, et al. [114] & 1200 & & Israel & $\begin{array}{c}\text { August } 15-\text { October } \\
5,2006\end{array}$ & Israel-Hezbollah War & $\begin{array}{l}\text { Cross-sectional; } \\
\text { telephone survey }\end{array}$ & PTSD & SCL \\
\hline $\begin{array}{c}\text { Papadopoulou, et } \\
\text { al. [56] }\end{array}$ & 5116 & & Greece & April 7 - May 3, 2020 & COVID-19 & $\begin{array}{l}\text { Cross-sectional; } \\
\text { online survey }\end{array}$ & $\begin{array}{l}\text { Anxiety, depression, } \\
\text { resilience }\end{array}$ & $\begin{array}{c}\text { GAD-2, PHQ-2, CD- } \\
\text { RISC2 }\end{array}$ \\
\hline Park, et al. [57] & 1015 & 38.9 & USA & April $7-9,2020$ & COVID-19 & $\begin{array}{l}\text { Cross-sectional; } \\
\text { online survey }\end{array}$ & $\begin{array}{c}\text { Coping, } \\
\text { General distress }\end{array}$ & $\begin{array}{l}\text { DERS-18, Brief } \\
\text { COPE, DASS-21, }\end{array}$ \\
\hline $\begin{array}{l}\text { Pat-Horenczyk, et al. } \\
\qquad(2006)\end{array}$ & 1336 & $\begin{array}{l}14.92 \\
(1.62)\end{array}$ & Israel & $2002-2003$ & Al Aqsa uprising & Cross-sectional & PTSD & $\begin{array}{c}\text { PTSD Child } \\
\text { Reaction Index - } \\
\text { Adolescent version }\end{array}$ \\
\hline Pollari, et al. [121] & 4934 & & USA & $\begin{array}{l}\text { T1: } 2003-2004 \\
\text { T2: } 2006-2007 \\
\text { T3: } 2011-2012 \\
\text { T4: } 2015-2016\end{array}$ & $\begin{array}{l}\text { Terrorist attacks of } \\
\qquad / 11\end{array}$ & Longitudinal & $\begin{array}{l}\text { Post-traumatic growth } \\
\text { stress }\end{array}$ & PTGI \\
\hline Prati, et al. [59] & 1569 & $\begin{array}{l}31.30 \\
(12.42)\end{array}$ & Italy & April, 2020 & COVID-19 & $\begin{array}{l}\text { Cross-sectional; } \\
\text { online survey }\end{array}$ & Mental health & GHQ-12 \\
\hline $\begin{array}{c}\text { Pérez-Fuentes, et } \\
\text { al. [58] }\end{array}$ & 1014 & $\begin{array}{c}40.87 \\
(12.42)\end{array}$ & Spain & & COVID-19 & Cross-sectional & Anxiety, depression & EVEA \\
\hline Ran, et al. [60] & 1770 & & China & $\begin{array}{l}\text { February } 23-\text { March } \\
\qquad 2,2020\end{array}$ & COVID-19 & Cross-sectional & $\begin{array}{l}\text { Resilience, depression, } \\
\text { anxiety }\end{array}$ & $\begin{array}{l}\text { CD-RISC, PHQ-9, } \\
\text { GAD-7 }\end{array}$ \\
\hline Ren, et al. [61] & 1172 & & China & $\begin{array}{l}\text { February } 14-\text { March } \\
\qquad 29,2020\end{array}$ & COVID-19 & Cross-sectional & $\begin{array}{c}\text { Depression, anxiety, } \\
\text { stress, resilience, PTSD }\end{array}$ & $\begin{array}{c}\text { PHQ-9, GAD-7, } \\
\text { PSS-10, CD-RISC10, } \\
\text { PCL-5 }\end{array}$ \\
\hline Riehm, et al. [96] & 6008 & & USA & $\begin{array}{c}\text { T1: March } 10-31 \text {, } \\
2020 \\
\text { T2: April } 1-\text { August 4, } \\
2020\end{array}$ & COVID-19 & $\begin{array}{l}\text { Longitudinal; } \\
\text { online survey }\end{array}$ & $\begin{array}{l}\text { Resilience, mental } \\
\text { distress }\end{array}$ & BRS, PHQ-4 \\
\hline $\begin{array}{l}\text { Rodriguez-Rey, et al. } \\
\text { (2020) }\end{array}$ & 3055 & $\begin{array}{c}32.15 \\
(12.95)\end{array}$ & Spain & March $17-24,2020$ & COVID-19 & Cross-sectional & Psycho-logical distress & IES-R \\
\hline Ryu, et al. [63] & 1500 & & South Korea & April 24 - May 5, 2020 & COVID-19 & Cross-sectional & Stress & PSS-10 \\
\hline Saxon, et al. [134] & 3600 & & $\begin{array}{l}\text { Republic of } \\
\text { Georgia }\end{array}$ & & $\begin{array}{c}\text { Armed conflicts in } \\
\text { the Rep. of Georgia in } \\
1992 \text { and } 2008\end{array}$ & Cross-sectional & $\begin{array}{l}\text { PTSD, depression, } \\
\text { anxiety, coping }\end{array}$ & $\begin{array}{l}\text { TSQ, PHQ-9, GAD-7, } \\
\text { Brief COPE }\end{array}$ \\
\hline Schäfer, et al. [82] & 1591 & $\begin{array}{c}55.03 \\
(13.90)\end{array}$ & $\begin{array}{l}\text { Germany, } \\
\text { Austria, } \\
\text { Switzerland }\end{array}$ & $\begin{array}{l}\text { T1: February } 17-23 \text {, } \\
2020 \\
\text { T2: March } 16-22 \\
2020\end{array}$ & COVID-19 & Longitudinal & Traumatic distress & PDI \\
\hline Schmitt, et al. [64] & 3274 & & Brazil & & COVID-19 & $\begin{array}{l}\text { Cross-sectional; } \\
\text { online survey }\end{array}$ & Depression, resilience & PHQ-9; CD-RISC \\
\hline Scott, et al. [122] & 2240 & & USA & 3 years following $9 / 11$ & $\begin{array}{l}\text { Terrorist attacks of } \\
9 / 11\end{array}$ & Longitudinal & PTSD, general distress & $\begin{array}{c}\text { IES-R, PCL, SCL-25, } \\
\text { BSI-18 }\end{array}$ \\
\hline Serafim, et al. [65] & 3000 & 39.8 & Brazil & May 22 - June 5, 2020 & COVID-19 & $\begin{array}{l}\text { Cross-sectional; } \\
\text { online survey }\end{array}$ & $\begin{array}{l}\text { Depression, anxiety, } \\
\text { coping }\end{array}$ & DASS-21, CSI \\
\hline Serrão, et al. [66] & 2008 & $38(10)$ & Portugal & May 9 - June 8, 2020 & COVID-19 & $\begin{array}{l}\text { Cross-sectional; } \\
\text { online survey }\end{array}$ & $\begin{array}{l}\text { Resilience, depression, } \\
\text { burnout }\end{array}$ & RS, DASS-21, CBI \\
\hline Shalhub, et al. [67] & 1609 & & 58 countries & April $14-24,2020$ & COVID-19 & $\begin{array}{l}\text { Cross-sectional; } \\
\text { online survey }\end{array}$ & Anxiety, coping & GAD-7, Brief COPE \\
\hline $\begin{array}{c}\text { Shoshani, et al. } \\
\text { [123] }\end{array}$ & 1078 & $\begin{array}{l}13.74 \\
(0.84)\end{array}$ & Israel & & $\begin{array}{l}\text { Israeli-Palestinian } \\
\text { coonflict }\end{array}$ & Cross-sectional & $\begin{array}{l}\text { Psycho-logical distress, } \\
\text { depression }\end{array}$ & BSI-18 \\
\hline Silver, et al. [124] & 3496 & & USA & $\begin{array}{l}\text { T1: Septem-ber } 20 \text { - } \\
\text { October 4, } 2001 \\
\text { T2: Novem-ber } 10- \\
\text { December 3, } 2001 \\
\text { T3: } 6 \text { months after the } \\
\text { attacks }\end{array}$ & $\begin{array}{l}\text { Terrorist attacks of } \\
9 / 11\end{array}$ & $\begin{array}{l}\text { Longitudinal; } \\
\text { online survey }\end{array}$ & Coping, stress & Brief COPE, SASRQ \\
\hline Sinawi, et al. [68] & 1538 & & Oman & March - April, 2020 & COVID-19 & $\begin{array}{l}\text { Cross-sectional; } \\
\text { online survey }\end{array}$ & Anxiety, depression & GAD-7, PHQ-9 \\
\hline Solomon, et al. [125] & 349 & & Israel & 1991, 2003, 2008, 2014 & Yom Kippur War & Longitudinal & PTSD & $\mathrm{PCL}$ \\
\hline Stene, et al. [139] & 281 & $19.3(4.3)$ & Norway & $\begin{array}{c}\text { T1: } 4-5 \text { months after the } \\
\text { attack } \\
\text { T2: } 14-15 \text { months after } \\
\text { the attack }\end{array}$ & Uttoya attack & Longitudinal & PTSD, mental distress, & $\begin{array}{l}\text { UCLA PTSD-RI, } \\
\text { SCL-8 }\end{array}$ \\
\hline
\end{tabular}




\begin{tabular}{|c|c|c|c|c|c|c|c|c|}
\hline Su, et al. [129] & 102 & $25.4(3.7)$ & Taiwan & $\begin{array}{c}\text { a one-month study } \\
\text { during a 7-week } \\
\text { period ending June 30, } \\
2003\end{array}$ & SARS & Longitudinal & $\begin{array}{l}\text { PTSD, depression, } \\
\text { anxiety }\end{array}$ & DTS, BDI, STAI \\
\hline Sweeny, et al. [69] & 5115 & $\begin{array}{l}21.36 \\
(4.39)\end{array}$ & China & February $12-19,2020$ & COVID-19 & $\begin{array}{l}\text { Cross-sectional; } \\
\text { online survey }\end{array}$ & Depression, anxiety & BSI \\
\hline Tsai, et al. [132] & & & USA & 2000,2001 & $\begin{array}{c}\text { Terrorist attacks of } \\
9 / 11\end{array}$ & Cross-sectional & Mental health & HRQOL \\
\hline Taylor, et al. [70] & 6854 & $49.8(16.2)$ & USA, Canada & March 21 - April 1, 2020 & COVID-19 & $\begin{array}{c}\text { Cross-sectional; } \\
\text { online survey }\end{array}$ & Anxiety, depression & PHQ-4 \\
\hline $\begin{array}{c}\text { Updegraff, et al. } \\
\text { [126] }\end{array}$ & 931 & & USA & $\begin{array}{l}\text { T1: Septem-ber } 20 \text { - } \\
\text { October 4, } 2001 \\
\text { T2: Novem-ber } 10- \\
\text { December 3, } 2001 \\
\text { T3: Septem-ber } 20- \\
\text { October 4, } 2002 \\
\text { T4: Septem-ber } 20 \text { - } \\
\text { October 4, } 2003\end{array}$ & $\begin{array}{c}\text { Terrorist attacks of } \\
9 / 11\end{array}$ & Longitudinal & Stress, coping, PTS & $\begin{array}{c}\text { SASRQ, Brief COPE, } \\
\text { PCL }\end{array}$ \\
\hline Varma, et al. [71] & 1653 & & $\begin{array}{l}63 \text { countries, } \\
\text { worldwide }\end{array}$ & $\begin{array}{l}\text { April } 9 \text { and May 25, } \\
2020\end{array}$ & COVID-19 & $\begin{array}{l}\text { Cross-sectional; } \\
\text { Online survey }\end{array}$ & $\begin{array}{l}\text { Depression, anxiety, } \\
\text { stress, resilience }\end{array}$ & $\begin{array}{l}\text { PHQ-9, STAI, PSS, } \\
\text { BRCS }\end{array}$ \\
\hline Veldhuis, et al. [103] & 1567 & & $\begin{array}{l}\text { USA, 50+ } \\
\text { countries }\end{array}$ & $\begin{array}{c}\text { T1: April } \\
5-19, \\
2020 \\
\text { T2: August } \\
28-\text { Septem-ber } \\
11,2020\end{array}$ & COVID-19 & $\begin{array}{l}\text { Longitudinal; } \\
\text { online survey }\end{array}$ & $\begin{array}{l}\text { Depression,stress, } \\
\text { anxiety }\end{array}$ & CES-D, IES, GAD-7 \\
\hline $\begin{array}{l}\text { Veronese, et al. } \\
\text { (2017) }\end{array}$ & 1276 & $8.8(1.42)$ & Palestine & $\begin{array}{l}\text { Two months after } \\
\text { military operation "Pillar } \\
\text { of Defense" ended }\end{array}$ & $\begin{array}{l}\text { Israeli military } \\
\text { peration "Pillar of } \\
\text { Defense" }\end{array}$ & Cross-sectional & $\begin{array}{l}\text { Positive and negative } \\
\text { affect, traumatic } \\
\text { response }\end{array}$ & PANAS-C, CRIES-13 \\
\hline $\begin{array}{c}\text { Veronese, et al. } \\
\text { (2019) }\end{array}$ & 143 & $\begin{array}{l}12.02 \\
(2.05)\end{array}$ & Palestine & $\begin{array}{c}\text { T1: January } 2018 \\
\text { T2: June - Septem-ber } \\
2018\end{array}$ & $\begin{array}{l}\text { Palestinian - Israeli } \\
\text { conflict }\end{array}$ & Longitudinal & Traumatic response & CRIES-13 \\
\hline Wang, et al. [149] & 1210 & & China & $\begin{array}{c}\text { January } 31 \text { - February } \\
2,2020\end{array}$ & COVID-19 & $\begin{array}{l}\text { Cross-sectional; } \\
\text { online survey }\end{array}$ & $\begin{array}{l}\text { Psycho-logical impact, } \\
\text { depression, anxiety, } \\
\text { stress }\end{array}$ & IES-R, DASS-21 \\
\hline Wang, et al. [149] & 1599 & & China & $\begin{array}{c}\text { February } \\
1-4 \\
2020\end{array}$ & COVID-19 & Cross-sectional & $\begin{array}{c}\text { Psycho-logical distress, } \\
\text { coping }\end{array}$ & K6, SCSQ \\
\hline Welch, et al. [127] & 17062 & & USA & $\begin{array}{l}\text { T1: } 2003-2004 \\
\text { T2: } 2006-2007 \\
\text { T3: } 2011-2012\end{array}$ & $\begin{array}{c}\text { Terrorist attacks of } \\
9 / 11\end{array}$ & Longitudinal & PTSD & PCL \\
\hline Yang, et al. [74] & 1638 & & China & February $1-4,2020$ & COVID-19 & $\begin{array}{l}\text { Cross-sectional; } \\
\text { online survey }\end{array}$ & Stress, coping & PSS-10, SCSQ \\
\hline Yan, et al. [73] & 3233 & $\begin{array}{l}31.71 \\
(9.78)\end{array}$ & China & $\begin{array}{c}\text { January } 31 \text { - February } \\
9,2020\end{array}$ & COVID-19 & $\begin{array}{l}\text { Cross-sectional; } \\
\text { online survey }\end{array}$ & Stress, coping & PSS-10, SCSQ \\
\hline Yan, et al. [73] & 3088 & $37.5(13.5)$ & China & February, 2020 & COVID-19 & Cross-sectional & Resilience & CD-RISC10 \\
\hline Ye, et al. [99] & 7800 & $\begin{array}{l}20.54 \\
(2.11)\end{array}$ & China & $\begin{array}{c}\text { January } 31-\text { February } \\
11,2020\end{array}$ & COVID-19 & $\begin{array}{c}\text { Cross-sectional; } \\
\text { online survey }\end{array}$ & Resilience & CD-RISC10 \\
\hline Yu, et al. [75] & 1588 & $\begin{array}{c}33.68 \\
(11.96)\end{array}$ & China & $\begin{array}{c}\text { February } 1 \text {-February } \\
4,2020\end{array}$ & COVID-19 & $\begin{array}{l}\text { Cross-sectional; } \\
\text { online survey }\end{array}$ & $\begin{array}{c}\text { Psycho-logical distress, } \\
\text { coping }\end{array}$ & K6, SCSQ \\
\hline $\begin{array}{l}\text { Zager Kocjan, et } \\
\text { al. [76] }\end{array}$ & 2722 & $\begin{array}{c}36.40 \\
(13.10)\end{array}$ & Slovenia & $\begin{array}{l}\text { five } \\
\text { days } \\
\text { after } \\
\text { Slovenia } \\
\text { declared } \\
\text { the } \\
\text { covID-19 } \\
\text { epidemics }\end{array}$ & COVID-19 & Cross-sectional & Coping, stress & CD-RISC10, PSS \\
\hline Zarrouq, et al. [102] & 1435 & & Morocco & April $3-30,2020$ & COVID-19 & Cross-sectional & Coping, depression & Brief COPE, HADS \\
\hline Zhou, et al. [150] & 442 & $\begin{array}{c}45.3 \\
(16.46)\end{array}$ & USA & April - May, 2020 & COVID-19 & Longitudinal & $\begin{array}{l}\text { Depression, anxiety, } \\
\text { stress, PT growth }\end{array}$ & DASS-21, PTGI \\
\hline Zoorob, et al. [77] & 1115 & & USA & April, 2020 & COVID-19 & Cross-sectional & $\begin{array}{l}\text { Burnout, depression, } \\
\text { resilience }\end{array}$ & RSWBI, BRS \\
\hline
\end{tabular}


Note. BDI: Beck Depression Inventory; BRCS: Brief Resilient Coping Scale; BRS: Brief Resilience Scale; BSI: Brief Symptom Inventory; CBI: Copenhagen Burnout Inventory; CDRISC: Connor-Davidson Resilience Scale; CDI: Child Depression Inventory; CDI: Children's Depression Inventory; CERQ-short: Short Cognitive Emotion Regulation Questionnaire; CES-D: Center for Epidemiological Studies-Depression Scale; CPTS-RI: Child Post-Traumatic Stress Reaction Index; CRIES: Children's Impact of event scale; CSI:Coping Strategies Inventory; CSQ: Coping Strategy Questionnaire; CSS: Coping Style Scale; CYRM: Child and Youth Resilience Measure; DASS: Depression, Anxiety and Stress Scale; DERS: Difficulties in Emotion Regulation Scale; DRC: Democratic Republic of Congo; DTS: Davidson Trauma Scale; EVEA: Mood Evaluation Scale; EWBI: expanded Well-being Index; GAD: Generalized Anxiety Disorder; GHQ: General Health Questionnaire; HADS: Hospital Anxiety and Depression Scale; HAS: Health Anxiety Scale; HEl: Huaxi EmotionalDistress Index; HRQOL: Health-Related Quality of Life; IES-R: Impact of Event Scale-Revised; ITQ: International Trauma Questionnaire; K6: Kessler Psychological Distress Scale; MBI-GS: Maslach Burnout Inventory-General Survey; MHC-SF: Mental Health Continuum; MINI: Mini-International Neuropsychiatric Interview; PANAS: Positive and Negative Affect Schedule; PAPF-PR: Parents' Assessment of Protective Factors Parental Resilience subscale; PC-PTSD: Primary Care-PTSD; PCL: PTSD Checklist; PDI: Peritraumatic Distress Inventory; PDS: Post-traumatic Diagnostic Scale; PHQ: Patient Health Questionnaire; PSS-SR: Post-traumatic Symptom Scale - Self-Report; PSS: Perceived Stress Scale; PTGI: Posttraumatic Growth Inventory; PTGI: Posttraumatic Growth Inventory; QPTSR-C: Questionnaire for Examination of Posttraumatic Stress Reactions in Children; QSTWE: Questionnaire on Children's Stressful and Traumatic War Experiences; RCMAS: The Revised Children's Manifest Anxiety Scale; RS: Resilience Scale; RSA: Resilience Scale for Adults; RSWBI: The Resident/Fellow Well-Being Index; SAS: Self-Rating Anxiety Scale; SASRQ: Stanford Acute Stress Reaction Questionnaire; SCAS: Spence Child Anxiety Scale; SCL: Symptom Check-List; SCSI-R: Revised School-Agers' Coping Strategies Inventory; SCSQ: Simplified Coping Style Questionnaire; SHAl: Short Health Anxiety Inventory; STAI: State-Trait Anxiety Inventory; SWEMWBS: Short Warwick Edinburgh Mental Wellbeing Scale; TSQ: Trauma Screening Questionnaire; UCLA PTSD-RI: University of California at Los Angeles PTSD Reaction Index; WCQ: Ways of Coping Questionnaire.

accident. The number of studies were relatively equally distributed over European (33\%), Asian (37\%), and American countries (22\%). Additionally, studies from Oceania (1\%) and Africa (1\%) were also represented, as well as studies spanning multiple countries and continents ( $5 \%)$. Most studies focused on the impact of disasters on the general public (62\%), but research on healthcare workers $(14 \%)$, specific age categories $(18 \%)$ (children, adolescents, elderly, etc.) and survivors of a disaster $(6 \%)$ were also included.

\section{Results of individual sources of evidence}

COVID-19 studies: Almost all studies included in this review pertain to the first few weeks of the pandemic and lockdowns [18-77]. For most countries in the world, lockdowns and relaxations went on for much longer and still continue today. Attention should be paid to the fact that the chronic character of COVID-19 and its impact on mental health is not yet accounted for in these studies. Moreover, different measures to combat the virus were taken across the globe which in their own right may impact mental health differently. However, it was recently demonstrated that findings from specific countries can be generalized across international borders [78].

Overall, the studies pertaining to the first weeks of lockdown report a decline in mental health in the general population $[16,20,22,25-30,32,33,35-41,43,45,50,51,53,54,56,58,61,65-$ $70,74,76,78-82]$. More specifically, high anxiety [16,2 $2,28,30,36,40,46,53,62,76]$ and depressive symptoms $[16,27,38,43,45,51,58,61-63,83-85]$ were reported in most studies. Furthermore, a substantial number of individuals scored above clinical cut-off points for both depression and anxiety [68], as well as for other mental disorders in general $[1,57]$. Moreover, high levels of stress and psychological distress were found $[18,31,32,47,55,57,68,70,71,73,74]$. Posttraumatic stress scores [46,57] and suicidal thoughts [56] increased in the general population. Other studies described a decrease in sleep duration and quality, which was found to negatively impact anxiety and depression [71,81]. The main worries that negatively impacted mental health for individuals during those first few weeks were the difficulty of obtaining daily necessities and concerns about harming others [63]. Since most studies were cross-sectional, it is not possible to compare the mental health data with any other point in time: even among the longitudinal studies, only one study included data gathered before and after the start of the pandemic. This study found, that mental health was stable overall, but that significant changes were observed in $20 \%$ of the individuals with increases or decreases in well-being compared to before the lockdown [82]. Interestingly, one study was carried out at the same time in different countries which were at different stages of lockdown and relaxations in the measures taken to control the pandemic. The authors demonstrated that in the first few weeks of the pandemic there were no mental health differences across the different countries [71]. However, at a later stage of the pandemic, countries which were more affected also reported higher rates of mental health problems, such as anxiety and depression $[79,86]$.

Thirteen out of 87 COVID-19 studies collected data in multiple waves during the first few weeks or months of the pandemic $[42,46,58,65,82,83,86-94]$. In keeping with the results of the cross-sectional studies, these studies found increased anxiety, depression and stress scores at the beginning of the lockdown and the pandemic in general [42,46,58,65,82,83,86-94]. Furthermore, more weeks spent in lockdown and isolation corresponded with less well-being, as well as an increase in unhealthy behaviors like overeating or alcohol use [64,69,93] and even suicidal ideation [56]. However, many studies also found a decrease in anxiety and depression rates as time went by and the reopening of society was announced $[88,89,91,92,94]$. This decrease was even found to constitute a relative rapid return to pre-pandemic rates after relaxations $[89,90]$. One study reported dips in the daily mental health in relation to announcements of negative COVID-19 news [61]. However, the study by Fukase and colleagues [85], which was conducted during the second wave, found that depression scores increased steeply again at the start of the second wave, representing a return to similar levels of well-being as during the first wave.

People who came in very close contact with COVID-19 patients most often are healthcare workers. Shalhub and colleagues [67] found that this group feared infecting their own family and suffered from high levels of stress linked to lack of available equipment in the first few weeks. It is, therefore, not surprising that higher levels of anxiety and depression were found among healthcare workers [67]. Moreover, higher levels of PTSD and trauma symptoms were also reported [24]. More 
close contact with COVID-19 patients was related to lower wellbeing scores [24] Overall, it is reported that many healthcare workers had to work longer hours indicating high levels of fatigue and exhaustion, and even burnout $[86,54,66]$. Longer working hours were also found to be related to less sleep and lower sleep quality which, in turn, negatively impacted anxiety [55]. Additionally, Cai and colleagues [21] demonstrated that, among healthcare workers, less work experience correlated with lower well-being scores, while the most important protective factors for healthcare workers were resilience and social support.

Besides healthcare workers, other groups and factors were also associated with a poorer mental health. Being female, unmarried [42,56,75] unemployed [73], having smaller living quarters $([38,20]$ or living in more adverse economic circumstances $[48,85]$, as well as having a migration background [35], were all risk factors found to negatively impact well-being. Moreover, individuals with pre-existing health conditions, for example a chronic disease $[42,59,64]$ or a mental health diagnosis $[56,71]$, also reported lower well-being scores during the pandemic compared to the general population. Living with someone at risk for a COVID-19 infection [42] as well as a close relationship with an infected individual $[29,34]$ was equally reported to be a risk factor. Furthermore, following the news was related to more psychological stress $[88,59,74]$ and increased sleep problems [88]. The evidence concerning educational levels is contradictory. Some studies found that higher educational levels were related to lower well-being [65] while others found that lower educational levels were related to higher well-being $[35,40,64,95,96]$. See Table 2 for a clear overview of the different risk factors, vulnerability factors and protective factors.

Different age groups also responded differently to lockdowns and the pandemic. While older individuals are at greater risk for contracting COVID-19, their mental health appears to be less affect by the pandemic $[40,97]$ compared to younger people and students whose mental health seems to be more affected by the pandemic. International students who were not able to return home were even more affected than other students [32]. With regards to children, the evidence is not conclusive. The study by Achterberg and colleagues [87] reported that children are spared from distress while another study found children to have lower well-being [98]. For parents on the other hand, the first few weeks of the pandemic seemed to be really demanding as many of them had to work from home while children were also present. High levels of psychological distress as well as parenting-related exhaustion were reported $[49,87]$. Interestingly, younger people and women showed more rapid improvements in wellbeing when measures were relaxed again [83].

Although COVID-19 has a significant impact on mental health, as demonstrated by the results discussed above, many people still displaygreat resilience $[18,22,43,60,76,86,96,97,99]$ However, longer lockdowns correspond with less overall resilience [43]. At the individual level, greater resilience was related to higher well-being and lower stress $[55,76,100]$ Furthermore, individuals with high resilience show a more stable trajectory over the lockdowns compared to individuals with less resilience. In line with previous research, elderly adults seem to have more resilience overall compared to the general population [22].

The reviewed research studied different coping mechanisms used by individuals and their effectiveness at mitigating mental health complaints $[20,23,88,26,27,92,31,85,32,33,34,3$ $9,40,44,46,101,51,57,99,75]$ Having social support, a sense of togetherness and looking for social support was found to be the most effective way of coping with lockdowns and the pandemic [20,27,92,97,46,51,53,56,57,82] Moreover, a positive attitude towards the preventive measures taken to restrict the spread of the virus, trust in the government [35,52,56,59] as well as psychological flexibility [27,50] and grit [50] were related to greater well-being. Other positive coping strategies were religious coping $[20,56,102]$, mindfulness [20,57,69], actively seeking help [51], cognitive restructuring and wishful thinking [32], doing physical exercise [71], having a routine [40,95] and spending time cooking [22].

On the other hand, it was found that alcohol consumption and smoking increased during the pandemic as a coping mechanism [101,61,103]. Moreover, overeating [70] and social media use increased significantly [20]. For some individuals,

Table 2: Overview of all the vulnerability -, risk- and protective factors.

\begin{tabular}{|c|c|c|}
\hline Vulnerability factors & Risk factors & Protective factors \\
\hline Healthcare workers & Unemployment & Living together as a couple \\
\hline Females & Being close to an infected person & Social support \\
\hline Smaller living space & Following the news closely & Psychological flexibility \\
\hline Low social economic status & Low social support \\
\hline Migrants & \\
\hline Pre-existing chronic disease & \\
\hline Pre-existing mental health problems & \\
\hline Pre-existing addiction & \\
\hline Youngsters & \\
\hline Parents with young children & \\
\hline
\end{tabular}


this was a useful way to cope and self-regulate positive mood [92] while for others this resulted in excessive informationseeking behaviors and less effective coping [88,92]. Overall, avoidant coping was found to be related to more depression, anxiety, and loneliness $[27,42,45,53,83]$.

\section{Other disaster-related studies: Phases of disaster ma- nagement}

In the next paragraph, the non-COVID-19 studies will be structured according to the phases of disaster management [6] in order to extrapolate conclusions on the possible future impact on mental health. Some studies have data on different phases and will thus be mentioned several times in different sections. Since most disasters are unpredictable and unfold quickly while research tends to proceed more slowly, most of the studies in this review were conducted several months after a disaster, hence, providing information about the recovery phase [104-127] Accordingly, the initial phases of disasters are represented in far less studies.

Warning or threat phase: The warning or threat phase describes the time period before a disaster occurs, when there already is a recognition (threat) or a warning that a disaster may occur. This might, for example, be the case in politically unstable countries before a war breaks out. In a longitudinal study, data was collected during a first war, between wars and after a second war. It was found that between wars, the distress was significantly lower. However, due to the politically unstable environment and the uncertain conditions, high levels of anxiety were reported [104].

Impact phase: The impact phase describes the moment of the disaster itself and varies depending on the type of disaster. Only a few studies were able to gather data right at the start of a disaster. At the moment of impact, it was found that being closer to the disaster resulted in significantly more post-traumatic symptoms $[128,129]$ Moreover, for the SARS outbreak specifically, during the outbreak, nurses were found to have very high levels of anxiety, stress, fear, symptomatic depression, and insomnia $[129,130]$. The main source of anxiety for these nurses was the fact of being subjected to conflicting demands of not endangering family members whilst at the same time carrying out their duty providing healthcare [130].

Rescue or heroic phase: This phase refers to the time immediately after a disaster. Many studies report high levels of PTSD symptoms immediately after a disaster strikes $[122,124,125,127,131]$ as well as a decline in mental health [132] Different coping strategies were identified that could be used during this phase. Active coping was related to higher levels of well-being, as were having a large social network and religion as a coping mechanism [131,133,134] Drinking, on the other hand, was related to lower well-being levels [131,134] Similarly, (over)actively following the news about the disasters was related to greater distress [135]. Having a routine and continuing normal activities to the extent possible, for example going to work, reduces negative mental health consequences [136]. Overall, women [119,135,137], young adults [135], migrants [135], and individuals with pre-existing conditions
[137] experience more distress and PTSD as a result of the disaster [135]. For children, PTSD symptoms immediately after a disaster are related to the proximity to the disaster while later on this relationship diminishes [117]. Overall, it was found that younger children are better able to cope with a disaster compared to school-age children [138].

Remedy or honeymoon phase: This phase takes place rather soon after the disaster, when the initial shock has settled down and there is a lot of solidarity within the community. This phase is mentioned ephemerally in many studies, but it is rarely systematically studied. Only one study included in the current review really focuses on examines this phase. This study found that students who did volunteer work after a disaster were able to see greater future possibilities for their lives. Moreover, many of them decided to start a career in medicine due to their volunteer experience [115].

Inventory phase: Not unlike the remedy or honeymoon phase, only a few studies shed light on the inventory phase in which people way their experience against the - perceived or actual - available help and support. In this review it was found that there was a sharp increase in visits to primary and secondary healthcare after a disaster, although this care could not always be provided [139]. Similarly, it was reported that some individuals who met the diagnostic criteria for mental health problems did not seek help as it was simply not widely available [137]

Disillusionment phase: In this phase, the mental health of many individuals returns to normal, while others continue to suffer from negative effects. This can create a divide in the community. Some studies found that this might depend on whether effective coping occurs immediately after the disaster and also on the levels of distress already reported at the initial phase [131,135,133,134] Adults who used an avoidant coping style, emotional suppression and/or self-blame showed decreased well-being [131,134]. For individuals who experienced the disaster close by a more enduring negative impact on mental health is reported, while others who were at a greater distance from the disaster showed mitigation of mental health problems already at an earlier moment in time [140].

Recovery phase: Most people turn out to be very resilient after a disaster, and their mental health and stress levels return to normal $[104,115,119,122-124,127]$ However, this is not the case for everyone and, influenced by several factors, significant differences exist in the mental health of individuals over time [107]. For PTSD symptoms it was found that the decrease after a disaster is very rapid [119] and that optimism is a very important factor facilitating this decrease [107]. Even though mental health mostly returns to normal, it was found that even 60 years after a disaster higher levels of PTSD were reported for people who experienced the disaster compared to those who did not [111]. Also, some individuals showed delayed-onset PTSD, an effect which is not detectable immediately after the disaster [125]. This is also influenced by more indirect impacts of the disaster, such as reduction of household income, employment 
status, etc. [116]. Individuals who were diagnosed with both PTSD and major depressive episodes also experienced more trauma compared to individuals who were only diagnosed with either PTSD or major depressive disorder [120]. Workplace conditions can also contribute to the mitigation of mental health impacts. Employees with high role clarity and low-level role conflict were for example found to have lower stress levels after the disaster $[106,140]$. Moreover, trying to find meaning in the disaster [126] and feeling satisfied with life [138]was related to a better adjustment over time.

Specific target groups exist for which the mental health effects and their development deviate from those found in the general population. Police responders, for example, had the lowest rates of PTSD prevalence compared to all of the other responders [109]. For children, it was found that, when being compared with younger children, school-age children experience a greater impact on their mental health in the long term due to a disaster [113]. As in adults, PTSD symptoms in children decrease over time, but for some children these symptoms were still prevalent after quite some time [117]. Moreover, trauma from a disaster is related to poorer educational and occupational outcomes [112]. Older adults on the other hand, even though they may have experienced much more trauma throughout their life, show greater resilience, which protects them from mental health problems [110]. Consequences of a disaster in some cases are not exclusively negative. Post-traumatic growth has been documented among individuals who have experienced a disaster. The individuals who have experienced more distress during and right after the disaster are usually those who display more post-traumatic growth afterwards $[114,118,121]$. The more central the disaster was to an individual's life, the more post-traumatic growth is reported [108]

\section{Discussion}

This scoping review focused on identifying the impact of the COVID-19 pandemic on mental health, including what can still be expected in the months to come. Of the 3,557 articles identified for this review, only 128 were included. More than half of the excluded studies pertained to COVID-19. Many research papers on COVID-19 attempt to describe the impact of the pandemic, but the urgency of the matter led researchers to make compromises on quality. Therefore, the current review applied not only thematic criteria to determine eligibility but also took study design and sample size into consideration whilst defining the inclusion criteria. Accordingly, only the highest quality of evidence was included. This in order to avoid that the growing number of papers characterized by a lower level of evidence could create $d$ a significant interpretation bias.

For instance, a recurrent cross-sectional study conducted based on a brief mental health survey with few items could report good mental health for a certain region, whereas data on usage of mental healthcare or on waiting lists for that region could indicate poor mental health. By only including studies supported by the highest level of evidence through a thorough examination of the design, questionnaires and sample size; this scoping review provides solid insights on mental health impact while also having predictive value for possible scenarios for the upcoming months.

Overall, the results indicate a significant impact on mental health in the general population. These effects are more prominent when strict measures are in place in comparison to time periods with more lenient measures [78]. Furthermore, the chronicity of the pandemic has an important impact on mental health: symptoms increase [93,64,69], unhealthy behaviors [93,64,69] and suicidal ideation [56] become more common. When a pandemic arises, we can expect all involved to be at an increased risk of developing stress, anxiety, depressive symptoms, insomnia, anger, grief and fear [141]. Indeed, this was confirmed by the research results in this scoping review. Furthermore, symptoms of insomnia and an increase in unhealthy behavior patterns became apparent. It is noteworthy that these symptoms interact dynamically with their context which would indicate that these symptoms are part of an adaptation process rather than a sign of psychological diagnosis. This is in line with the theoretical model of conservation of resources [142]. This model assumes that people strive to maintain, protect, and build resources and that their sense of threat is related to the potential or actual loss of these valued resources. Research supports the hypothesis that current loss of psychosocial resources - for instance social interaction and the ability to continue a daily routine - contributes more to symptomatology than premorbid stressors [141]. Context is key in resilience and post-traumatic growth. Context includes the possibility of maintaining daily routines and roles. For instance, work routines characterized by a consistent pattern of attending work in a regular, predictable manner were found to have positive effects on mental health [136]. The results in this review indicate that females and younger people show more post-traumatic growth after a disaster [114,118,121]. When strict measures were relaxed more rapid improvements in well-being were already detectable among younger people and females [83]. These findings are in line with the theory that post-traumatic growth is more prevalent in individuals who initially struggled more [143].

The elderly are generally found to be more resilient than other age groups, although they are more prone to COVID-19. Their resilience also protects them from mental health problems [110]. This might be due to elderly people being more emotionally stable and having more life experience. Overall, it appears that coping strategies present and used at the moment when a disaster strikes have a significant impact on the level of resilience. Due to their life experiences, the elderly might already know which coping strategies will be most effective for them, while other age groups still need to come up with these effective strategies.

The use of different coping methods in reducing psychological distress differed across studies. Conflicting results could be explained in consideration of the individual's appraisal of the situation. Lazarus and Folkman [144]. suggested that a 'goodness of fit' has to be achieved between the individual's appraisal of the situation and the coping strategy selected in order to maximize its effectiveness. The use 
of problem-focused strategies predicts better adjustment in controllable situations while emotion-focused strategies such as optimism are favorable in uncontrollable situations [144]. Returning to the elderly who displayed greater resilience, this could indicate that older adults who may have more experience dealing with health issues and strict measures appraised the situation as more controllable, which perhaps resulted in more resilience due to using the best-fitting coping strategy. Coping could also explain the wide range of effects on mental health. The results in this review indicate coping as a leverage for more positive outcomes [110].

Policymakers should consider the varying impact of the pandemic on mental health and focus on modifiable factors to foster resilience and post-traumatic growth. In targeting atrisk groups, policymakers should focus on risk factors rather than on age groups given the fact that large variations within age groups exist $[78,145]$ and in addition, also take into account the cumulative and multiplicative effect of these risk factors $[78,145]$.

Healthcare workers (HCW) are particularly at risk of traumatic symptoms because of the highly stressful situations to which they are exposed during their work, including management of critical medical situations, frequent witnessing of death and trauma, operating in crowded settings, interrupted circadian rhythm due to shift work [2] as well as the risk of contaminating family with contagions acquired while working [2,67]. HCW include not only medical HCW but also psychosocial care workers $[78,145]$. Interestingly, research examining the impact of traumatogenic events on healthcare workers describing the immediate impact and even potential delayed effects on their mental health exists, however, there is no data available that would enable us to predict what can be expected in the aftermath as monitoring tends to stop immediately after the event (Carmassi et al., 2020). Young people also require special attention as they seem to be more prone to mental distress. Other risk factors for a longterm, negative impact on well-being include a pre-existing (mental) health condition, isolation, low social support, poorer living conditions, pre-occupation with COVID-related news, unemployment or fear of job loss, or close encounters with cases of Covid-19. Younger people and females were also identified as being more at risk. Vulnerability factors include pre-existing mental health problems, healthcare profession or migration background. Fortunately, there are some protective factors, such as cohabitation with a partner, quality of social contacts and family well-being. These factors are multiplicative and cumulative: when multiple life areas are affected, the risk of long-term distress increases along with its effects on daily life [78]. Previous research has reported PTSD rates to range from 10 to about $20 \%$ [2] with even higher rates in healthcare workers [2]. Although most individuals prove to be resilient after being exposed to a traumatic event, several risk factors may hinder effective adaptation, all of which are aligned with the factors mentioned above [2]. Experiencing feelings of helplessness or intensity of emotions when exposed to traumatic events influences the levels of resilience [2]. This again emphasizes the importance of the context for promoting a sense of control in order to alleviate feelings of helplessness or intense emotions.

The phases of response to disaster model provides insights into what can be expected during the upcoming months of the pandemic. The most significant finding is the importance of maintaining psychosocial monitoring measures, which must continue to be in place for at least 6 months after the pandemic. If a biomedical model were to be applied, pandemicspecific measures might be able to stop after the medical crisis phases. However, the extension of these supportive measures proves to be necessary in order to address the delayed onset of psychosocial problems such as delayed-onset PTSD $[2,78,129,130]$. Monitoring, detection, triage and referral to professional help when needed is, thereby, essential for providing the best possible care and helping people on their way to optimal resilience. This review clearly demonstrates that psychological crisis intervention can and should play a pivotal role in the implementation of comprehensive disease control and rehabilitation measures after a disaster. Clearly, however, this is not always the case: mental health care is not yet fully integrated within emergency preparedness, let alone pandemic preparedness. As Brewin and colleagues [146] demonstrate, the psychosocial dimension is, indeed, lacking in official pandemic plans around the world. While spontaneous and well-meaning mental health efforts do arise, these are rarely integrated into regular care, leading to different unforeseen, negative effects: lack of quality control, duplication, inconsistency in content, data collection and privacy risks, etc. Unfortunately, this constitutes a missed opportunity for effectively dealing with the pandemic in itself and the consequential mental health needs. As Cullen and colleagues [147] indicate, a prevalence of biomedical concerns is understandable in the immediate acute impact/heroic phase of a new outbreak, as health systems prioritize critical patient care and reducing transmission, but the crucial importance of the psychosocial aspects during a pandemic must never be overlooked. After all, psychosocial factors are also linked to level of adherence to containment measures and, therefore, crucial in stopping the spread of the virus itself. As Campion and colleagues [148-150] mention, the early detection of mental health needs in the general population and in specific target groups can help to implement actions needed to alleviate symptoms before they become problematic. It also ensures that a qualitatively adequate response can be implemented when and wherever necessary, instead of a one-size-fits-all approach which can often be ineffective, inefficient, or even superfluous.

This scoping review has several noteworthy strengths. First and foremost, only studies supported by the highest level of evidence were included to provide insights into the current impact of the pandemic on mental health as well as its possible future impact. Using these strict inclusion criteria avoids interpretation bias (above all by disregarding numerous papers published based on questionable designs and/or limited sample size, most of which are convenience samples). Another strength of this paper lies in its organization of findings using the theoretical model of phases of response to disaster. The model enables a structured presentation of a wide range of 
studies and topics while also permitting comparisons between different studies.

This main limitation of our scoping review is the limited availability of COVID-19 data at the time of research, which only exist for the period up to August 2020 and has been mostly collected during the first lockdowns. The phases of response to disaster model was used as a theoretical model in an attempt to compensate for this selection bias. Further follow up research is necessary in order to refine the model and cultivate further knowledge on the impact of a pandemic on mental health needs, especially in the months and years following the pandemic.

\section{Conclusion}

This scoping review reveals a dynamic interaction between the individuals' mental health and the context in which they live. Strict measures impacting normal life routines lead to less resilience, whereas more lenient measures which are more or less compatible with daily routines and the upholding of life roles lead to greater resilience in dealing with the pandemic. Several risk factors are identified which lead to a better understanding of the impact of the pandemic on mental health, thereby, providing policymakers with indicators that could enable them to adopt a more proactive approach. Furthermore, the phases of response to disaster model provides an understandable narrative, while also enabling society to make useful predictions about what to expect for the upcoming months. The most important consideration is the risk of delayed onset of psychosocial problems. This justifies the recommendation to policymakers to not only further invest the necessary efforts in order to manage the significant impact of the pandemic on mental health now, but also to continue to do so for the time to come.

\section{Acknowledgement}

E.V.H. and N.V.d.C contributed to the conception and design of the study.

E.V.H. developed the search strategy.

E.V.H., E.P., H.D.L., and S.H. contributed to the acquisition of data.

H.D.L. analyzed and interpreted the data.

E.V.H. and N.V.d.C. drafted the first version of the introduction of this manuscript.

E.V.H.and H.D.L. drafted the revised manuscript.

E.V.H. and H.D.L. read, provided feedback an approved the final revised manuscript.

\section{Author's note}

This article is partially established through collaboration with the voluntary expert group on COVID-19 and mental health from the Belgian Superior Health Council. No competing interests to declare.

\section{References}

1. Li Q (2020) Psychosocial and coping responses toward 2019 coronavirus diseases (COVID-19): a cross-sectional study within the Chinese general population. Qjm 113: 731-738. Link: https://bit.ly/3ynTBof

2. Carmassi C, Foghi C, Dell'Oste V, Cordone A, Bertelloni CA, et al. (2020) PTSD symptoms in healthcare workers facing the three coronavirus outbreaks: What can we expect after the COVID-19 pandemic. Psychiatry Research 292: Link: https://bit.ly/3221XFH

3. Walsh F (2007) Traumatic loss and major disasters: Strengthening family and community resilience. Family Process 46: 207-227. Link: https://bit.ly/3IME4TC

4. American Psychiatric Association (2013) Diagnostic and statistical manual of mental disorders (5th ed.). Link: https://bit.ly/3ET8GRa

5. Brooks SK, Webster RK, Smith LE, Woodland L, Wessely S, et al. (2020) The psychological impact of quarantine and how to reduce it: rapid review of the evidence. Lancet 395: 912-920. Link: https://bit.ly/3GFTvev

6. DeWolfe DJ (2000) Training Manual for Mental Health and Human Service Workers in Major Disasters. Rockville, MD: U.S. Department of Health and Human Services, Substance Abuse and Mental Health Services Administration, Center for Mental Health Services.

7. McMahon K (2011) The psychology of disaster. Peak Oil Blues.

8. May CL, Wisco BE (2016) Defining trauma: How level of exposure and proximity affect risk for posttraumatic stress disorder. Psychological Trauma: Theory, Research, Practice, and Policy 8: 233-240. Link: https://bit.ly/3pZjZ45

9. Boin A, 't Hart P, Stern E, Sundelius B (2005) The Politics of Crisis Management: Public Leadership Under Pressure. Cambridge University Press. Link: https://bit.ly/3s5qqoW

10. Nohrstedt D, Bynander F, Parker C, 't Hart P (2018) Managing Crises Collaboratively: Prospects and Problems-A Systematic Literature Review. Perspectives on Public Management and Governance 1: 257-271. Link: https://bit.ly/3oSeS6f

11. Jordan AJ, Turnpenny JR (2015) The tools of policy formulation: Actors, capacities, venues and effects. The Tools of Policy Formulation: Actors, Capacities, Venues and Effects 1-320. Link: https://bit.ly/3m0gucs

12. Raphael B (1986) When disaster strikes: How individuals and communities cope with catastrophe. New York: Basic Books.

13. Raphael B, Newman L, Silove D, Bryant R, Boman B, et al. (2000) Disaste Mental Health Response Handbook. In The NSW Institute of Psychiatry. Link: https://bit.ly/3INTUxn

14. Gründer-Fahrer S, Schlaf A, Wiedemann G, Heyer G (2018) Topics and topical phases in German social media communication during a disaster. In Natural Language Engineering 24. Link: https://bit.ly/3EXvMG4

15. Baird ME (2010) The "Phases" of Emergency Management. Intermodal Freight Transportation Institute (IFTI). https://bit.ly/31ZQk2n

16. Waugh W (2000) Living with Hazards, Dealing with Disaster: An Introduction to Emergency Management,. Armonk, New York: M.E. Sharpe, Inc. Link: https://bit.ly/3dRGbH

17. Tricco AC, Lillie E, Zarin W, O'Brien KK, Colquhoun H, et al. (2018) PRISMA Extension for Scoping Reviews (PRISMA-ScR): Checklist and Explanation. Annals of Internal Medicine 169: 467-473. Link: https://bit.ly/3GNzYsN

18. Barzilay R, Moore TM, Greenberg DM, DiDomenico GE, Brown LA, et al. (2020) Resilience, COVID-19-related stress, anxiety and depression during the pandemic in a large population enriched for healthcare providers. Trans Psychiatry 10: 291. Link: https://bit.ly/3oORJBD

Citation: Van Hoof E, Laet HD, Hochrath S, Philips E, Horczak P, et al. (2021) Unravelling the impact of COVID-19 on mental health: A scoping review on traumatogenic events using the phases of response to disaster model. Arch Community Med Public Health 7(3): 218-237. DOI: https://dx.doi.org/10.17352/2455-5479.000165 
19. Budimir S, Probst T, Pieh C (2021) Coping strategies and mental health during COVID-19 lockdown. J Ment Health 30: 156-163. Link: https://bit.ly/3pSsAFr

20. Büssing A, Rodrigues Recchia D, Hein R, Dienberg T (2020) Perceived changes of specific attitudes, perceptions and behaviors during the Corona pandemic and their relation to wellbeing. Health Qual Life Outcomes 18: 374. Link: https://bit.ly/3m4n6GC

21. Cai W, Lian B, Song X, Hou T, Deng G, et al. (2020) A cross-sectional study on mental health among health care workers during the outbreak of Corona Virus Disease 2019. Asian J Psychiatr 51 : 102111. Link: https://bit.ly/3INmUoO

22. Carriedo A, Cecchini JA, Fernández-Río J, Méndez-Giménez A (2020) Resilience and physical activity in people under home isolation due to COVID-19: A preliminary evaluation. Mental Health and Physical Activity 19: 5. Link: https://bit.ly/3m5jLXP

23. Cauberghe V, Van Wesenbeeck I, De Jans S, Hudders L, Ponnet K (2020) How Adolescents Use Social Media to Cope with Feelings of Loneliness and Anxiety During COVID-19 Lockdown. Cyberpsychol Behav Soc Netw 24: 250 257. Link: https://bit.ly/3GEIIGX

24. Chen R, Sun C, Chen JJ, Jen HJ, Kang XL, et al. (2020) A Large-Scale Survey on Trauma, Burnout, and Posttraumatic Growth among Nurses during the COVID-19 Pandemic. Int J Ment Health Nurs 30: 102-116. Link: https://bit.ly/3yqUn3y

25. Chen SX, Ng JCK, Hui BPH, Au AKY, Wu WCH, et al. (2021) Dual impacts of coronavirus anxiety on mental health in 35 societies. Sci Rep 11: 8925. Link: https://bit.ly/3s0Nq8t

26. Cheng C, Lau YC, Luk JW (2021) Social Capital-Accrual, Escape-From-Self, and Time-Displacement Effects of Internet Use During the COVID-19 Stay-atHome Period: Prospective, Quantitative Survey Study. J Med Internet Res 22 e22740. Link: https://bit.ly/3ETYelV

27. Chong YY, Chien WT, Cheng HY, Lamnisos D, L̦ubenko J, et al. (2021) Patterns of Psychological Responses among the Public during the Early Phase of COVID-19: A Cross-Regional Analysis. Int J Environ Res Public Health 18. Link: https://bit.ly/3yqurAk

28. Dong ZQ, Ma J, Hao YN, Shen XL, Liu F, et al. (2020) The social psychologica impact of the COVID-19 pandemic on medical staff in China: A cross-sectional study. Eur Psychiatry 63: e65. Link: https://bit.ly/3IHFe2R

29. Duan H, Yan L, Ding X, Gan Y, Kohn N, et al. (2020) Impact of the COVID-19 pandemic on mental health in the general Chinese population: Changes, predictors and psychosocial correlates. Psychiatry Res 293: 113396.

30. Fernández RS, Crivelli L, Guimet NM, Allegri RF, Pedreira ME (2020) Psychological distress associated with COVID-19 quarantine: Latent profile analysis, outcome prediction and mediation analysis. J Affect Disord 277 75-84. Link: https://bit.ly/3oSfit

31. Fu W, Wang C, Zou L, Guo Y, Lu Z, et al. (2020) Psychological health, sleep quality, and coping styles to stress facing the COVID-19 in Wuhan, China TransI Psychiatry 10: 225. Link: https://bit.ly/3s4V9IG

32. Garbóczy S, Szemán-Nagy A, Ahmad MS, Harsányi S, Ocsenás D, Rekenyi V, et al. (2021) Health anxiety, perceived stress, and coping styles in the shadow of the COVID-19. BMC Psychol 9: 53. Link: https://bit.ly/31VVtbV

33. Gori A, Topino E, Di Fabio A (2020) The protective role of life satisfaction coping strategies and defense mechanisms on perceived stress due to COVID-19 emergency: A chained mediation model. PLoS One 15: e0242402. Link: https://bit.ly/3yqLMhF

34. Guo J, Feng XL, Wang XH, Van Ij MH (2020) Coping with COVID-19: Exposure to COVID-19 and Negative Impact on Livelihood Predict Elevated Mental Health Problems in Chinese Adults. Int J Environ Res Public Health 17. Link: https://bit.ly/31TB7jz

35. Harris SM, Al GM (2021) COVID-19 and psychological distress in Norway: The role of trust in the healthcare system. Scand J Public Health 49: 96-103. Link: https://bit.ly/3dRab6w

36. Hennein R, Mew EJ, Lowe SR (2021) Socio-ecological predictors of mental health outcomes among healthcare workers during the COVID-19 pandemic in the United States. PLoS One 16: e0246602. Link: https://bit.ly/3oTbyYt

37. Hou T, Zhang T, Cai W, Song X, Chen A, et al. (2020) Social support and mental health among health care workers during Coronavirus Disease 2019 outbreak: A moderated mediation model. PLoS One 15: e0233831. Link: https://bit.ly/3F6mGXy

38. Jacques-Aviñó C, López-Jiménez T, Medina-Perucha L, de Bont J, Gonçalves $A Q$, et al. (2020) Gender-based approach on the social impact and mental health in Spain during COVID-19 lockdown: a cross-sectional study. BMJ Open 10: e044617. Link: https://bit.ly/3yqUvQA

39. Jungmann SM, Witthöft M (2020) Health anxiety, cyberchondria, and coping in the current COVID-19 pandemic: Which factors are related to coronavirus anxiety? J Anxiety Disord 73: 102239. Link: https://bit.ly/31Zg278

40. Justo-Alonso A, García-Dantas A, González-Vázquez Al, Sánchez-Martín M, Del Río-Casanova L (2020) How did Different Generations Cope with the COVID-19 Pandemic? Early Stages of the Pandemic in Spain. Psicothema 32: 490-500. Link: https://bit.ly/3GIWSI2

41. Kavčič T, Avsec A, Zager Kocjan G (2020) Psychological Functioning of Slovene Adults during the COVID-19 Pandemic: Does Resilience Matter? Psychiatr Q 92: 207-216. Link: https://bit.ly/3dRvd4I

42. Kazan Kızılkurt O, Yılmaz A, Noyan CO, Dilbaz N (2020) Health anxiety during the early phases of COVID-19 pandemic in Turkey and its relationship with postpandemic attitudes, hopelessness, and psychological resilience. Perspect Psychiatr Care 57: 399-407. Link: https://bit.ly/3IOrd3u

43. Kimhi S, Marciano H, Eshel Y, Adini B (2020b) Resilience and demographic characteristics predicting distress during the COVID-19 crisis. Soc Sci Med 265: 113389. Link: https://bit.ly/3mp70Yv

44. Lahiri A, Jha SS, Acharya R, Dey A, Chakraborty A (2021) Has loneliness and poor resilient coping influenced the magnitude of psychological distress among apparently healthy Indian adults during the lockdown? Evidence from a rapid online nation-wide cross-sectional survey. PLoS One 16: e0245509. Link: https://bit.ly/3GIGeSp

45. Le Vigouroux S, Goncalves A, Charbonnier E (2021) The Psychological Vulnerability of French University Students to the COVID-19 Confinement. Health Educ Behav 48: 123-131. Link: https://bit.ly/3m5kDeZ

46. Li Y, Peng J (2021) Does social support matter? The mediating links with coping strategy and anxiety among Chinese college students in a crosssectional study of COVID-19 pandemic. BMC Public Health 21: 1298. Link: https://bit.ly/31VVOvd

47. Luceño-Moreno L, Talavera-Velasco B, García-Albuerne $Y$, Martín-García J (2020) Symptoms of Posttraumatic Stress, Anxiety, Depression, Levels of Resilience and Burnout in Spanish Health Personnel during the COVID-19 Pandemic. Int J Environ Res Public Health 17. Link: https://bit.ly/33tyLIF

48. Makhashvili N, Javakhishvili JD, Sturua L, Pilauri K, Fuhr DC, et al. (2020) The influence of concern about COVID-19 on mental health in the Republic of Georgia: a cross-sectional study. Global Health 16: 111. Link: https://bit.ly/3GL8aF4

49. Marchetti D, Fontanesi L, Mazza C, Gi D, Omenico S, et al. (2020) Parentingrelated exhaustion during the Italian COVID-19 lockdown. Journal of Pediatric Psychology 45: 1114-1123. Link: https://bit.ly/3DNrGz2

50. McCracken LM, Badinlou F, Buhrman M, Brocki KC (2021) The role of psychological flexibility in the context of COVID-19: Associations with depression, anxiety, and insomnia. Journal of Contextual Behavioral Science 19: 28-35. Link: https:/bit.ly/3EOhONO

Citation: Van Hoof E, Laet HD, Hochrath S, Philips E, Horczak P, et al. (2021) Unravelling the impact of CoVID-19 on mental health: A scoping review on traumatogenic events using the phases of response to disaster model. Arch Community Med Public Health 7(3): 218-237. DOI: https://dx.doi.org/10.17352/2455-5479.000165 
51. McFadden P, Ross J, Moriarty J, Mallett J, Schroder H, et al. (2021) The Role of Coping in the Wellbeing and Work-Related Quality of Life of UK Health and Social Care Workers during COVID-19. Int J Environ Res Public Health 18. Link: https://bit.ly/3IRsyX1

52. Mechili EA, Saliaj A, Kamberi F, Girvalaki C, Peto E, et al. (2021) Is the mental health of young students and their family members affected during the quarantine period? Evidence from the COVID-19 pandemic in Albania. $J$ Psychiatr Ment Health Nurs 28: 317-325. Link: https://bit.ly/3oRIPDm

53. Minahan J, Falzarano F, Yazdani N, Siedlecki KL (2021) The COVID-19 Pandemic and Psychosocial Outcomes Across Age Through the Stress and Coping Framework. Gerontologist 61: 228-239. Link: https://bit.ly/31Zgmmm

54. Mohd Fauzi MF, Mohd Yusoff H, Muhamad Robat R, Mat Saruan NA, Ismail KI, et al. (2020) Doctors' Mental Health in the Midst of COVID-19 Pandemic: The Roles of Work Demands and Recovery Experiences. Int J Environ Res Public Health 17. Link: https://bit.ly/3oRIVLe

55. Mosheva M, Hertz-Palmor N, Dorman Ilan S, Matalon N, Pessach IM, Afek A, et al. (2020) Anxiety, pandemic-related stress and resilience among physicians during the COVID-19 pandemic. Depress Anxiety 37: 965-971. Link: https://bit.ly/3EOhseY

56. Papadopoulou A, Efstathiou V, Yotsidi V, Pomini V, Michopoulos I, et al. (2021) Suicidal ideation during COVID-19 lockdown in Greece: Prevalence in the community, risk and protective factors. Psychiatry Res 297: 113713. Link: https://bit.ly/3e5BVo9

57. Park CL, Finkelstein-Fox L, Russell BS, Fendrich M, Hutchison M, et al. (2021) Americans' distress early in the COVID-19 pandemic: Protective resources and coping strategies. Psychol Trauma 13 : 422-431. Link: https://bit.ly/3DV29UK

58. Pérez-Fuentes MDC, Molero Jurado MDM, Martos Martínez Á, Gázquez Linares JJ (2020) Threat of COVID-19 and emotional state during quarantine: Positive and negative affect as mediators in a cross-sectional study of the Spanish population. PLoS One 15: e0235305. Link: https://bit.ly/3DQ5gNz

59. Prati G (2020) Mental health and its psychosocial predictors during national quarantine in Italy against the coronavirus disease 2019 (COVID-19). Anxiety Stress Coping 34: 145-156. Link: https://bit.ly/3s3b9EV

60. Ran L, Wang W, Ai M, Kong Y, Chen J, et al. (2020) Psychological resilience, depression, anxiety, and somatization symptoms in response to COVID-19: A study of the general population in China at the peak of its epidemic. Soc Sci Med 262: 113261. Link: https://bit.ly/3INUGdL

61. Ren Y, Qian W, Li Z, Liu Z, Zhou Y, et al. (2020) Public mental health under the long-term influence of COVID-19 in China: Geographical and temporal distribution. J Affect Disord 277: 893-900. Link: https://bit.ly/3p0925a

62. Rodríguez-Rey R, Garrido-Hernansaiz H, Collado S (2020) Psychological impact of COVID-19 in Spain: Early data report. Psychol Trauma 12: 550-552. Link: https://bit.ly/3dQWxA8

63. Ryu S, Park IH, Kim M, Lee YR, Lee J, et al. (2021) Network study of responses to unusualness and psychological stress during the COVID-19 outbreak in Korea. PLoS One 16: e0246894. Link: https://bit.ly/3m65g6d

64. Schmitt AA, Brenner J, de Carvalho Alves AM, Claudino L, de A, et al. (2021) Potential predictors of depressive symptoms during the initial stage of the COVID-19 outbreak among Brazilian adults. Journal of Affective Disorders 282: 1090-1095. Link: https://bit.ly/3GHygJ9

65. Serafim AP, Durães RSS, Rocca CCA, Gonçalves PD, Saffı F, et al. (2021) Exploratory study on the psychological impact of COVID-19 on the general Brazilian population. PLoS One 16: e0245868. Link: https://bit.ly/3yqC9PV

66. Serrão C, Duarte I, Castro L, Teixeira A (2021) Burnout and Depression in Portuguese Healthcare Workers during the COVID-19 Pandemic-The Mediating Role of Psychological Resilience. Int J Environ Res Public Health 18. Link: https://bit.ly/3EUpCXv
67. Shalhub S, Mouawad NJ, Malgor RD, Johnson AP, Wohlauer MV, et al. (2020) Global vascular surgeons' experience, stressors, and coping during the coronavirus disease 2019 pandemic. J Vasc Surg 73: 762-771.e4. Link: https://bit.ly/3DRqquL

68. Sinawi HA, Al Balushi N, Al-Mahrouqi T, Al Ghailani A, McCall RK, et al. (2020) Predictors of psychological distress among the public in Oman amid coronavirus disease 2019 pandemic: a cross-sectional analytical study. Psychol Health Med 26: 131-144. Link: https://bit.ly/3IRsMgP

69. Sweeny K, Rankin K, Cheng X, Hou L, Long F, et al. (2020) Flow in the time of COVID-19: Findings from China. PLoS One 15: e0242043. Link: https://bit.ly/3EP3Lk5

70. Taylor SL, Ry CA, Paluszek MM, Fergus TA, McKay D, et al. (2020) COVID stress syndrome: Concept, structure, and correlates. Depress Anxiety 37: 706-714. Link: https://bit.ly/3pTvXMo

71. Varma $P$, Junge $M$, Meaklim H, Jackson ML (2020) Younger people are more vulnerable to stress, anxiety and depression during COVID-19 pandemic: A global cross-sectional survey. Prog Neuropsychopharmacol Biol Psychiatry 109: 110236. Link: https://bit.ly/3F1IK7a

72. Wang H, Xia Q, Xiong Z, Li Z, Xiang W, et al. (2020) The psychological distress and coping styles in the early stages of the 2019 coronavirus disease (COVID-19) epidemic in the general mainland Chinese population: A webbased survey. PLoS One 15: e0233410. Link: https://bit.ly/33nfhFr

73. Yan S, Xu R, Stratton TD, Kavcic V, Luo D, et al. (2021) Sex differences and psychological stress: responses to the COVID-19 pandemic in China. BMC Public Health 21: 79. Link: https://bit.ly/3s3bhnT

74. Yang X, Xiong Z, Li Z, Li X, Xiang W, et al. (2020) Perceived psychological stress and associated factors in the early stages of the coronavirus disease 2019 (COVID-19) epidemic: Evidence from the general Chinese population. PLoS One 15: e0243605. Link: https://bit.ly/3yt10aO

75. Yu H, Li M, Li Z, Xiang W, Yuan Y, Liu Y, et al. (2020) Coping style, social support and psychological distress in the general Chinese population in the early stages of the COVID-19 epidemic. BMC Psychiatry 20: 426. Link: https://bit.ly/3GIGP6B

76. Zager Kocjan G, Kavčič T, Avsec A (2020) Resilience matters: Explaining the association between personality and psychological functioning during the COVID-19 pandemic. International Journal of Clinical and Health Psychology 21: 9. Link: https://bit.ly/3EVbKw2

77. Zoorob D, Shah S, La Saevig D, Murphy C, Aouthmany S, et al. (2021) Insight into resident burnout, mental wellness, and coping mechanisms early in the COVID-19 pandemic. PLoS One 16: e0250104. Link: https://bit.ly/30ogvPE

78. Van Hoof Elke De Laet H, Résibois M, Gérard S, Dekeyser S, Loix E, et al. (2021) Living Document I: Belgian mental health (care) data repository. Zenodo.

79. Cénat JM, Dalexis RD, Guerrier M, Noorishad PG, Derivois D, et al. (2021) Frequency and correlates of anxiety symptoms during the COVID-19 pandemic in low- and middle-income countries: A multinational study. J Psychiatr Res 132: 13-17. Link: https://bit.ly/33iXU8G

80. Moya-Lacasa C, Alvarez-Vázquez CM, González-Blanco L, Valtueña-García M Martín-Gil E, et al. (2021) Impact of the coronavirus outbreak on mental health in the different Spanish regions. Actas Esp Psiquiatr 49: 64-70.

81. Rodrigues S, Paiva J, Dias D, Aleixo M, Filipe R, et al. (2018) Cognitive Impact and Psychophysiological Effects of Stress Using a Biomonitoring Platform. International Journal of Environmental Research and Public Health 15: 1080. Link: https://bit.ly/3DNsgNi

82. Schäfer SK, Sopp MR, Schanz CG, Staginnus M, Göritz AS, et al. (2020) Impact of COVID-19 on Public Mental Health and the Buffering Effect of a Sense of Coherence. Psychother Psychosom 89 : 386-392. Link: https://bit.ly/3yrvC7K

Citation: Van Hoof E, Laet HD, Hochrath S, Philips E, Horczak P, et al. (2021) Unravelling the impact of CoVID-19 on mental health: A scoping review on traumatogenic events using the phases of response to disaster model. Arch Community Med Public Health 7(3): 218-237. DOI: https://dx.doi.org/10.17352/2455-5479.000165 
83. Fancourt D, Steptoe A, Bu F (2020) Trajectories of anxiety and depressive symptoms during enforced isolation due to COVID-19 in England: a longitudinal observational study. Lancet Psychiatry 8: 141-149. Link: https://bit.ly/31WvW1Y

84. Hou WK, Lee TM, Liang L, Li TW, Liu H, et al. (2021) Psychiatric symptoms and behavioral adjustment during the COVID-19 pandemic: evidence from two population-representative cohorts. Transl Psychiatry 11: 174. Link: https://bit.ly/3DVwUIW

85. Fukase $Y$, Ichikura K, Murase H, Tagaya H (2021) Depression, risk factors and coping strategies in the context of social dislocations resulting from the second wave of COVID-19 in Japan. BMC Psychiatry 21: 33. Link: https://bit.ly/3pYjuXK

86. Banerjee S, Lim KHJ, Murali K, Kamposioras K, Punie K, et al. (2021) The impact of COVID-19 on oncology professionals: results of the ESMO Resilience Task Force survey collaboration. ESMO Open 6: 100058. Link: https://bit.ly/3dLNmRE

87. Achterberg M, Dobbelaar S, Boer OD, Crone EA (2021) Perceived stress as mediator for longitudinal effects of the COVID-19 lockdown on wellbeing of parents and children. Sci Rep 11: 2971. Link: https://bit.ly/3s5rUzw

88. Cheng C, Ebrahimi OV, Lau YC (2021) Maladaptive coping with the infodemic and sleep disturbance in the COVID-19 pandemic. J Sleep Res 30: e13235. Link: https://bit.ly/3IJMOFn

89. Daly M, Robinson E (2021) Longitudinal changes in psychological distress in the UK from 2019 to September 2020 during the COVID-19 pandemic: Evidence from a large nationally representative study. Psychiatry Res 300 : 113920. Link: https://bit.ly/3s2leSD

90. Daly M, Robinson E (2021) Psychological distress and adaptation to the COVID-19 crisis in the United States. J Psychiatr Res 136: 603-609. Link: https://bit.ly/3yD2E4V

91. Yan L, Gan Y, Ding X, Wu J, Duan H (2021) The relationship between perceived stress and emotional distress during the COVID-19 outbreak: Effects of boredom proneness and coping style. J Anxiety Disord 77: 102328. Link: https://bit.ly/3pUx1zN

92. Fluharty M, Bu F, Steptoe A, Fancourt D (2020) Coping strategies and mental health trajectories during the first 21 weeks of COVID-19 lockdown in the United Kingdom. Soc Sci Med 279: 113958. Link: https://bit.ly/3GJmON8

93. Kimhi S, Eshel Y, Marciano H, Adini B (2020a) A Renewed Outbreak of the COVID-19 Pandemic: A Longitudinal Study of Distress, Resilience, and Subjective Well-Being. Int J Environ Res Public Health 17. Link: https://bit.ly/3DY8ZIR

94. Lorenzo NE, Zeytinoglu S, Morales S, Listokin J, Almas AN, et al. (2021) Transactional Associations Between Parent and Late Adolescent Internalizing Symptoms During the COVID-19 Pandemic: The Moderating Role of Avoidant Coping. J Youth Adolesc 50: 459-469. Link: https://bit.ly/3p09yQE

95. Hou WK, Tong $\mathrm{H}$, Liang L, Li TW, Liu H, et al. (2021) Probable anxiety and components of psychological resilience amid COVID-19: A population-based study. J Affect Disord 282 : 594-601. Link: https://bit.ly/3pVb8QF

96. Riehm KE, Brenneke SG, Adams LB, Gilan D, Lieb K, et al. (2020) Association between psychological resilience and changes in mental distress during the COVID-19 pandemic. J Affect Disord 282: 381-385. Link: https://bit.ly/3EZIVj1

97. Li F, Luo S, Mu W, Li Y, Ye L, et al. (2021) Effects of sources of social support and resilience on the mental health of different age groups during the COVID-19 pandemic. BMC Psychiatry 21: 16. Link: https://bit.ly/3oVDU4x

98. Duan L, Shao X, Wang Y, Huang Y, Miao J, et al. (2020) An investigation of mental health status of children and adolescents in china during the outbreak of COVID-19. J Affect Disord 275: 112-118. Link: https://bit.ly/322cZv3
99. Ye Z, Yang X, Zeng C, Wang Y, Shen Z, et al. (2020) Resilience, Social Support, and Coping as Mediators between COVID-19-related Stressful Experiences and Acute Stress Disorder among College Students in China. Appl Psychol Health Well Being 12: 1074-1094. Link: https://bit.ly/3s892Qm

100. Mikocka-Walus A, Stokes M, Evans S, Olive L, Westrupp E (2021) Finding the power within and without: How can we strengthen resilience against symptoms of stress, anxiety, and depression in Australian parents during the COVID-19 pandemic? J Psychosom Res 145: 110433. Link: https://bit.ly/3dSeM8p

101. Martínez-Cao C, de la Fuente-Tomás L, Menéndez-Mir Al, Velasco Á, ZurrónMadera P, et al. (2021) Factors associated with alcohol and tobacco consumption as a coping strategy to deal with the coronavirus disease (COVID-19) pandemic and lockdown in Spain. Addict Behav 121: 107003. Link: https://bit.ly/31Wnq3n

102. Zarrouq B, Abbas N, Hilaly JE, Asri AE, Abbouyi S, et al. (2021) An investigation of the association between religious coping, fatigue, anxiety and depressive symptoms during the COVID-19 pandemic in Morocco: a web-based cross-sectional survey. BMC Psychiatry 21: 264. Link: https://bit.ly/3GJ09z0

103. Veldhuis CB, Nesoff ED, McKowen ALW, Rice DR, Ghoneima H, et al. (2021) Addressing the critical need for long-term mental health data during the COVID-19 pandemic: Changes in mental health from April to September 2020. Prev Med 146: 106465. Link: https://bit.ly/3IR7nEM

104. Ben-Zur H, Gilbar O (2011) Resilience and distress: Israelis respond to the disengagement from Gaza and the second Lebanese war. Community Ment Health J 47: 551-559. Link: https://bit.ly/3DT94h3

105. Birkeland Skogbrott M, Hafstad GS, Blix I, Heir T (2014) Latent classes of posttraumatic stress and growth. Anxiety, Stress \& Coping: An International Journal 28: 272-286. Link: https://bit.ly/326CWJP

106. Birkeland SM, Nielsen MB, Knardahl S, Heir T (2015) Associations between work environment and psychological distress after a workplace terror attack: the importance of role expectations, predictability and leader support. PLoS One 10: e0119492. Link: https://bit.ly/3yr5ART

107. Birkeland Skogbrott M, Blix I, Solberg $\varnothing$, Heir T (2016) Does optimism act as a buffer against posttraumatic stress over time? A longitudinal study of the protective role of optimism after the 2011 Oslo bombing. Psychologica Trauma: Theory, Research, Practice, and Policy 9: 207-213. Link: https://bit.ly/3GNRCfE

108. Blix I, Birkel Skogbrott M, Hansen MB, Heir T (2015) Posttraumatic growth and centrality of event: A longitudinal study in the aftermath of the 2011 Oslo bombing. Psychological Trauma: Theory, Research, Practice, and Policy 7: 18-23. Link: https://bit.ly/3dUPG94

109. Bowler RM, Han H, Gocheva V, Nakagawa S, Alper H, et al. (2010) Gender differences in probable posttraumatic stress disorder among police responders to the 2001 World Trade Center terrorist attack. Am J Ind Med 53: 1186-1196. Link: https://bit.ly/323fz3E

110. Eshel Y, Kimhi S, Lahad M, Leykin D (2016) Individual, Community, and National Resiliencies and Age: Are Older People Less Resilient than Younger Individuals? Am J Geriatr Psychiatry 24: 644-647. Link: https://bit.ly/3ysLnej

111. Freitag S, Braehler E, Schmidt S, Claesmer H (2013) The impact of forced displacement in World War II on mental health disorders and health-related quality of life in late life-A German population-based study. International Psychogeriatrics 25 : 310-319. Link: https://bit.ly/3oXH9IQ

112. Hadi F, Lai BS, Llabre MM (2014) Life outcomes influenced by war-related experiences during the Gulf crisis. Anxiety Stress Coping 27: 156-175 Link: https://bit.ly/3oVM64P

Citation: Van Hoof E, Laet HD, Hochrath S, Philips E, Horczak P, et al. (2021) Unravelling the impact of CoVID-19 on mental health: A scoping review on traumatogenic events using the phases of response to disaster model. Arch Community Med Public Health 7(3): 218-237. DOI: https://dx.doi.org/10.17352/2455-5479.000165 
113. Halevi G, Djalovski A, Vengrober A, Feldman R (2016) Risk and resilience trajectories in war-exposed children across the first decade of life. J Child Psychol Psychiatry 57: 1183-1193. Link: https://bit.ly/3IO5YyH

114. Hobfoll SE, Canetti-Nisim D, Johnson RJ, Palmieri PA, Varley JD, et al (2008) The association of exposure, risk, and resiliency factors with PTSD among Jews and Arabs exposed to repeated acts of terrorism in Israel. Journal of Traumatic Stress 21: 9-21. Link: https://bit.ly/3m5a37M

115. Kaye-Kauderer HP, Levine J, Takeguchi Y, Machida M, Sekine H, et al. (2019) Post-Traumatic Growth and Resilience Among Medical Students After the March 2011 Disaster in Fukushima, Japan. Psychiatr Q 90: 507-518. Link: https://bit.ly/3EXudbr

116. Kung WW, Liu X, Goldmann E, Huang D, Wang X, et al. (2018) Posttraumatic stress disorder in the short and medium term following the World Trade Center attack among Asian Americans. Journal of Community Psychology 46: 1075-1091. Link: https://bit.ly/326CUlb

117. Kuterovac-Jagodić G (2003) Posttraumatic stress symptoms in Croatian children exposed to war: a prospective study. J Clin Psychol 59: 9-25. Link: https://bit.ly/3dTXvM0

118. Levine SZ, Laufer A, Stein E, Hamama-Raz Y, Solomon Z (2009) Examining the relationship between resilience and posttraumatic growth. J Trauma Stress 22: 282-286. Link: https://bit.ly/3DXMOTp

119. Matt GE, Vázquez C (2008) Anxiety, depressed mood, self-esteem, and traumatic stress symptoms among distant witnesses of the 9/11 terrorist attacks: transitory responses and psychological resilience. Span J Psychol 11: 503-515. Link: https://bit.ly/3GJPUvT

120. Morina N, Ajdukovic D, Bogic M, Franciskovic T, Kucukalic A, et al. (2013) Co-occurrence of major depressive episode and posttraumatic stress disorder among survivors of war: how is it different from either condition alone? J Clin Psychiatry 74: e212- e218. Link: https://bit.ly/31YImqu

121. Pollari CD, Brite J, Brackbill RM, Gargano LM, Adams SW, et al. (2021) World Trade Center Exposure and Posttraumatic Growth: Assessing Positive Psychological Change 15 Years after 9/11. Int J Environ Res Public Health 18: 104. Link: https://bit.ly/3s88Xfw

122. Scott SB, Poulin MJ, Silver RC (2013) A lifespan perspective on terrorism: age differences in trajectories of response to 9/11. Dev Psychol 49: 986998. Link: https://bit.ly/3dYPCEZ

123. Shoshani A, Slone M (2016) The resilience function of character strengths in the face of war and protracted conflict. Frontiers in Psychology 6: 10 Link: https://bit.ly/326CCL7

124. Silver RC, Holman EA, Mclntosh DN, Poulin M, Gil-Rivas V (2002) Nationwide longitudinal study of psychological responses to September 11. JAMA 288 1235-1244. Link: https://bit.ly/3q10JTy

125. Solomon Z, Bachem R, Levin Y, Crompton L, Ginzburg K (2018) Long-term trajectories of posttraumatic stress disorder: Categorical versus continuous assessment. Psychiatry: Interpersonal and Biological Processes 81: 376390. Link: https://bit.ly/3ES79L3

126. Updegraff JA, Silver RC, Holman EA (2008) Searching for and finding meaning in collective trauma: results from a national longitudinal study of the 9/11 terrorist attacks. J Pers Soc Psychol 95: 709-722. Link: https://bit.ly/3oWeDqJ

127. Welch $A E$, Caramanica $K$, Maslow $C B$, Brackbill RM, Stellman SD, et al. (2016) Trajectories of PTSD Among Lower Manhattan Residents and Area Workers Following the 2001 World Trade Center Disaster 2003-2012. J Trauma Stress 29: 158-166. Link: https://bit.ly/3GM3DCm

128. Hamama-Raz Y, Solomon Z, Cohen A, Laufer A (2008) PTSD symptoms, forgiveness, and revenge among Israeli Palestinian and Jewish adolescents. J Trauma Stress 21: 521-529. Link: https://bit.ly/3IUL3Ku
129. Su TP, Lien TC, Yang CY, Su YL, Wang JH, et al. (2007) Prevalence of psychiatric morbidity and psychological adaptation of the nurses in a structured SARS caring unit during outbreak: A prospective and periodic assessment study in Taiwan. Journal of Psychiatric Research 41: 119-130. Link: https://bit.ly/3dQf9Av 6

130. Koh D, Lim MK, Chia SE, Ko SM, Qian F, et al. (2005) Risk Perception and Impact of Severe Acute Respiratory Syndrome (SARS) on Work and Personal Lives of Healthcare Workers in Singapore: What Can We Learn? Medical Care 43: 676-682. Link: https://bit.ly/3IUeRX

131. Butler LD, Koopman C, Azarow J, Blasey CM, et al. (2009) Psychosocial predictors of resilience after the September 11, 2001 terrorist attacks. Journal of Nervous and Mental Disease 197: 266-273. Link: https://bit.ly/3yq8Mx4

132. Tsai AC, Venkataramani AS (2015) Communal bereavement and resilience in the aftermath of a terrorist event: Evidence from a natural experiment. Soc Sci Med 146: 155-163. Link: https://bit.ly/3oT9olw

133. Mclntosh DN, Poulin MJ, Silver RC, Holman EA (2011) The distinct roles of spirituality and religiosity in physical and mental health after collective trauma: a national longitudinal study of responses to the $9 / 11$ attacks. $J$ Behav Med 34: 497-507. Link: https://bit.ly/3GNGG1B

134. Saxon L, Makhashvili N, Chikovani I, Seguin M, McKee M, et al. (2017) Coping strategies and mental health outcomes of conflict-affected persons in the Republic of Georgia. Epidemiol Psychiatr Sci 26: 276-286. Link: https://bit.ly/3q0Moqa

135. Cardeña E, Dennis JM, Winkel M, Skitka LJ (2008) A snapshot of terror: acute posttraumatic responses to the September 11 attack. J Trauma Dissociation 6: 69-84. Link: https://bit.ly/3e8IZjT

136. Biron M, Link S (2013) Stress, appraisal and work routine in wartime: Do men and women differ? Anxiety, Stress \& Coping: An International Journal 27: 229-240. Link: https://bit.ly/3pQQKAg

137. DeLisi LE, Maurizio A, Yost M, Papparozzi CF, Fulchino C, et al. (2003) A survey of New Yorkers after the Sept. 11, 2001, terrorist attacks. Am J Psychiatry 160: 780-783. Link: https://bit.ly/3EYCBHC

138. Veronese G, Pepe A, Jaradah A, Al Muranak F, Hamdouna H (2016) Modelling life satisfaction and adjustment to trauma in children exposed to ongoing military violence: An exploratory study in Palestine. Child Abuse Negl 63: 61-72. Link: https://bit.ly/3dOQwEe

139. Stene LE, Dyb G (2015) Health service utilization after terrorism: longitudinal study of survivors of the 2011 Utøya attack in Norway. BMC Health Serv Res 15: 158. Link: https://bit.ly/325PQaY

140. Nissen A, Birkel Nielsen M, Solberg Ø, Bang Hansen M, Heir T (2015) Perception of threat and safety at work among employees in the Norwegian ministries after the 2011 Oslo bombing. Anxiety Stress Coping 28: 650662. Link: https://bit.ly/3ITejB2

141. Chew QH, Wei KC, Vasoo S, Sim K (2020) Psychological and Coping Responses of Health Care Workers Toward Emerging Infectious Disease Outbreaks: A Rapid Review and Practical Implications for the COVID-19 Pandemic. Singapore Med J 61: 3. Singapore Med J 61: 350-356. Link: https://bit.ly/3oWdlfC

142. Hobfoll SE (1989) Conservation of Resources: A New Attempt a Conceptualizing Stress. American Psychologist 44: 513-524. Link: https://bit.ly/3ysKT7Z

143. Tedeschi RG, Calhoun LG (2004) Psychological Inquiry Posttraumatic Growth: A Developmental Perspective. Psychological Inquiry 15: 1-18. Link https://bit.ly/3q4Ik8G

144. Lazarus RS, Folkman S (1984) Stress, Appraisal, and Coping. Springer Publishing Company. Link: https://bit.ly/3pVq69

Citation: Van Hoof E, Laet HD, Hochrath S, Philips E, Horczak P, et al. (2021) Unravelling the impact of CoVID-19 on mental health: A scoping review on traumatogenic events using the phases of response to disaster model. Arch Community Med Public Health 7(3): 218-237. DOI: https://dx.doi.org/10.17352/2455-5479.000165 
145. Van Hoof E, Godderis L, Van leuven F, Van den Cruyce N, Bal S, et al. (2021a) Psychosocial care during the covid-19 pandemic revision. Link: https://bit.ly/3DR1AeF

146. Brewin CR, DePierro J, Pirard P, Vazquez C, Williams R (2020) Why we need to integrate mental health into pandemic planning. Perspectives in Public Health 140: 309-310. Link: https://bit.ly/3IUeuMr

147. Cullen W, Gulati G, Kelly BD (2020) Mental health in the COVID-19 pandemic. Qjm: An International Journal of Medicine 113: 311-312. Link: https://bit.ly/3EYCtl8
148. Campion J, Javed A, Sartorius N, Marmot M (2020) Addressing the public mental health challenge of COVID-19. The Lancet Psychiatry 7: 657-659. Link: https://bit.ly/3GKo1E5

149. Wang C, Pan R, Wan X, Tan Y, Xu L, et al. (2020) Immediate Psychological Responses and Associated Factors during the Initial Stage of the 2019 Coronavirus Disease (COVID-19) Epidemic among the General Population in China. Int J Environ Res Public Health 17: 1729. https://bit.ly/3yqpSuS

150. Zhou Y, MacGeorge EL, Myrick JG (2020) Mental Health and Its Predictors during the Early Months of the COVID-19 Pandemic Experience in the United States. Int J Environ Res Public Health 17. Link: https://bit.ly/31Rh5GA

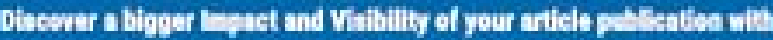 Peertechr Publications}

\section{HighWhis}

- Sipnatsry putininer af OMOD

- Sphatsy Putisher of DCFA CBan Francisos Detbates an Research Assessmetín

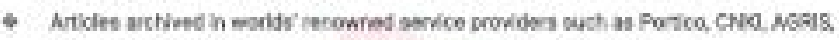

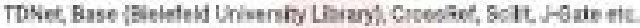

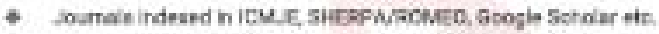

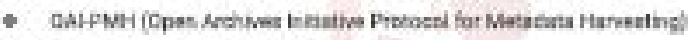

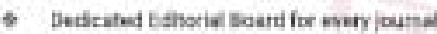

- Acturate and repid peer review prosess

- hereasad etobions of putishad articles through promobisns

- Reduoed tmelhe for article pibloation

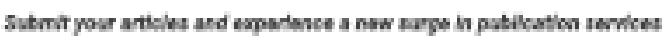

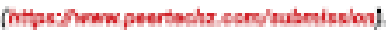

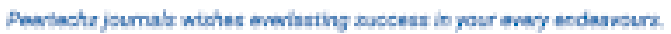

Citation: Van Hoof E, Laet HD, Hochrath S, Philips E, Horczak P, et al. (2021) Unravelling the impact of COVID-19 on mental health: A scoping review on traumatogenic events using the phases of response to disaster model. Arch Community Med Public Health 7(3): 218-237. DOI: https://dx.doi.org/10.17352/2455-5479.000165 